\title{
étude du comportement élastique et fragile des roches saturées par un liquide
}

\section{F.-H. Cornet}

Physicien-Adjoint à I'Institut de Physique du Globe de Paris

\section{ETUDE DU COMPORTEMENT ELASTIQUE ET FRAGILE DES ROCHES SATUREES PAR UN LIOUIDE}

L'objet de cet article est de discuter de l'applicabilité des principes de la mécanique des milieux continus à l'étude des roches poreuses et saturées par un liquide.

Une nouvelle définition de la porosité est proposée pour caractériser les variations de porosité dans l'espace rocheux considéré. Cette définition est utilisée pour dériver l'équation d'équilibre des roches saturées et discuter les différents modèles de comportement élastique linéaire proposés précédemment pour ces matériaux. Ces résultats ont été vérifiés expérimentalement sur un grès et un calcaire ; ils se sont révélés satisfaisant pour le calcaire mais non applicable au grès, celui-ci pré sentant un comportement élastique fortement non linéaire. Du fait de cette non linéarité, une nouvelle définition de la limite de comportement élastique a été proposée.

Le comportement post-élastique de ces deux roches, c'est-à-dire de leur désintégration, a été étudié expérimentalement par des essais triaxiaux drainés effectués avec une presse doublement servo-asservie (de 30 bars à 500 bars de pression de confinement). Il a été observé que pour certaines valeurs de la pression de confinement effective la roche présentait une dilatance négative, ou contractance, phénomène qui était associé à un comportement instable vis-à-vis du travail des forces extérieures. II est donc proposé de distinguer deux types de dilatance:

- la dilatance irréversible associée au développement de microfissures ;

- la dilatance réversible associée à un comportement élastique non linéaire.

Les différentes notions de contrainte effective ont été discutées; seule la théorie classique de Terzaghi (1923) s'est révélée satisfaisante et ce uniquement lorsque certaines hypothèses simplificatrices sont vérifiées.

Finalement l'influence de la vitesse de déformation est discutée et une nouvelle technique d'essais pour les roches saturées est proposée qui permettra de lever les limitations imposées actuellement par les conditions de drainage.

\section{ANALYSIS OF THE ELASTIC BRITTLE BEHAVIOR OF SATURATED POROUS ROCKS}

The purpose of this article is to discuss the applicability of continuum mechanics principles to the analysis of the mechanical behavior of saturated porous rocks.

A new definition of rock porosity is proposed which provides a mean for characterizing porosity variations in a given rock space. This definition is used first to derive equilibrium equations in saturated porous rocks, secondly to discuss the various theory which have been proposed previously for the analysis of the linearly elastic response of these rocks. The validity of the results have been investigated experimentally on Indiana limestone and Berea sandstone. It has been found that whilst Indiana limestone exhibits a linearly elastic behavior. Berea sandstone is strongly non-linear. Because of this lack of linearity a new definition for the limits of the elastic domaine, derived from energy considerations, has been proposed.

The post elastic behavior of these two rocks. i.e. the desintegration process, was investigated experimentally by servo-controlled drained triaxial tests (confining pressure ranging from 30 bars to 500 bars). It has been observed that for some domaine of effective confining pressures, both rocks exhibited a negative dilatancy, called contractancy; this contractancy was associated with an unstable behavior with respect to the work of external forces for Berea sandstone and a stable one in the case of Indiana limestone. These observations support the hypothesis that two kinds of dilatancy should be considered:

- an irreversible dilatancy associated with the development of microfissures;

- a reversible dilatancy associated with a nonlinear elastic response.

Finally the various effective stress concepts have been discussed; only that proposed by Terzaghi in 1923 was found reasonable, and this only when some simplifying assumptions are valid. In this context, the strain rate influence has been discussed; a new testing procedure for saturated rocks has been proposed which removes part of the testing constraints normally imposed by the drainage conditions. 
Comité Français de Mécanique des Roches 


\title{
étude du comportement élastique et fragile des roches saturées par un liquide
}

\author{
par F.-H. CORNET
}

\section{INTRODUCTION}

La géométrie excessivement compliquée, tant de la matrice solide que des pores qui constituent une roche, rend illusoire la détermination exacte des mouvements d'un point quelconque à l'intérieur d'un volume rocheux lorsque celui-ci subit une déformation. Aussi, la roche est-elle généralement assimilée à un matériau continu équivalent dont on essaie de déterminer les caractéristiques de façon que son comportement mécanique soit aussi proche que possible de celui de la roche.

L'objet de cet exposé est de discuter de l'applicabilité des principes de la mécanique des milieux continus à l'étude des matériaux intrinsèquement hétérogènes, discontinus et biphasiques que sont les roches saturées.

Tout d'abord, la notion de porosité est discutée et la loi d'équilibre dynamique pour le matériau continu équivalent est dérivée dans le cas où la porosité est isotrope et où le liquide s'écoule selon la loi de Darcy.
La théorie de l'élasticité linéaire appliquée aux milieux poreux est ensuite présentée et les théories de Biot $(1941,1955)$ et de Lubinski (1954) sont discutées. L'analyse de mesures expérimentales sur le grès de Berea et le calcaire de l'Indiana permet alors de préciser le domaine de validité de ces lois ainsi que la notion de limite élastique.

Puis la description des résultats d'essais triaxiaux drainés, effectués au moyen d'une presse servo-asservie sur les deux roches précédemment mentionnées, permet d'aborder le problème de la dilatance ainsi que celui de la résistance des roches saturées et drainées.

Finalement, la notion de contrainte effective est discutée et le problème de la modélisation du comportement "post-élastique ", non quasi-statique, des roches saturées brièvement abordé.

\section{EQUATIONS D'EQUILIBRE}

La dérivation des équations d'équilibre pour une roche saturée requiert tout d'abord que le rapport géométrique solide-liquide soit explicité.

\subsection{Etude de la porosité}

La porosité est définie traditionnellement soit de façon volumique, soit de façon surfacique, soit encore de façon linéaire (voir, par exemple, Scheideger 1964). Cependant, ces définitions classiques ne sont pas complètement satisfaisantes car elles ne permettent pas de représenter les variations possibles de porosité dans l'espace considéré ; elles devraient donc être modifiées.

Considérons deux points $x$ et $y$ à l'intérieur d'un volume rocheux; on peut définir la porosité linéaire du vecteur $\underline{x}-\underline{y}=\underline{u}$ par la relation :

$$
1(\underline{x}, \underline{u})=\frac{\left|\underline{u^{p}}\right|}{|\underline{u}|}
$$

où $\left|u^{p}\right|$ est la portion de $\underline{u}$ qui recoupe l'espace poreux.

$$
\text { Si } u=0 \text { : }
$$

$1(x, \overline{0})=0$ si $x$ appartient à la matrice solide;

$1(\bar{x}, \overline{0})=1$ si $\underline{x}$ appartient à l'espace poreux.

Si l'on représente les variations, quand $|u|$ augmente, de $1(x, u)$ pour un point $x$ situé à l'intérieur de la matrice solide et pour un point $x^{\prime}$ situé dans l'espace poreux au voisinage immédiat de $x$, on obtient des courbes du type de celles représentées sur la figure 1 . Pour une valeur de $u$ suffisamment grande les valeurs de $1(x, u)$ et de $1\left(x^{\top}, u\right)$ deviennent très proches l'une de l'autre.
Fig. 1. - Variations de la porosité linéaire en fonction du module du vecteur $\mathbf{u}$.

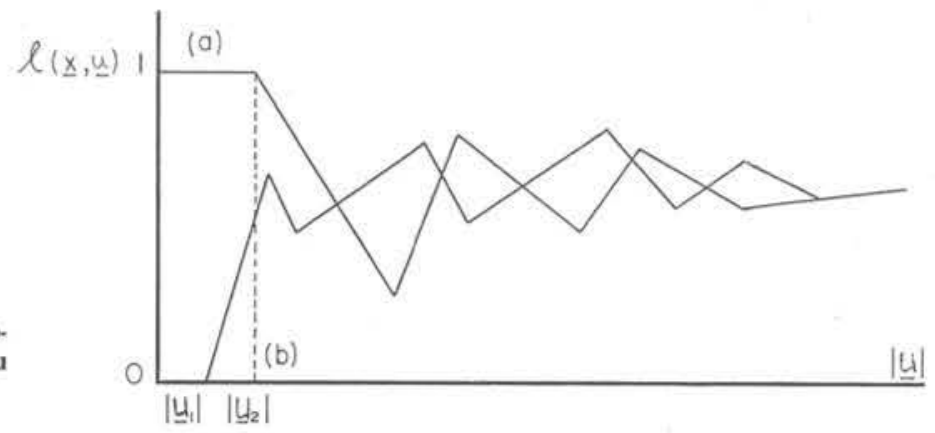


Ainsi on peut définir la porosité linéaire en un point $x$ d'une roche dans la direction $n$ ( $n$ vecteur unitaire) par la relation :

$$
1=1\left(\underline{x}, \underline{u}_{0}\right)
$$

où $\underline{u}_{0}=k \underline{n}$ est défini par la relation :

$$
\left|1\left(\underline{x}, \underline{u}_{0}\right)-1\left(\underline{x^{\prime}}, \underline{u_{0}}\right)\right|<\varepsilon
$$

$\varepsilon$ est choisi en relation avec la précision requise. Il est bien évident que pour des valeurs de $\varepsilon$ infiniment petites, le vecteur $u_{0}$ ne peut être défini; on choisira généralement $\varepsilon$ de l'ordre de 0.005 . Lorsque la porosité linéaire est indépendante de la direction de $n$ elle est dite isotrope; c'est le cas lorsque les pores sont distribués de façon quelconque.

On peut maintenant définir la porosité de surface de la roche considérée par la relation :

$$
f=f\left(\underline{x}, a_{0} \underline{n}\right)=\frac{a_{0}^{p}}{a_{0}}
$$

où $n$ est la normale à la surface plane d'aire $a_{0}$ définie en coordonnées polaires par la relation :

$$
a_{0}=\int_{0}^{2 \pi} \frac{1}{2}\left|u_{0}\right|^{2} d \theta
$$

avec :

$$
a_{0}^{p}=\int_{0}^{2 \pi} \frac{1}{2} l\left(\underline{x}, \underline{u}_{0}\right) \underline{u}_{0} \cdot \underline{u}_{0} d \theta
$$

Raisonnant en coordonnées polaires, on supposera que $u_{0}$ dépend de $\underline{x}$ et de $\theta$ lorsque $\underline{n}$ est donné.

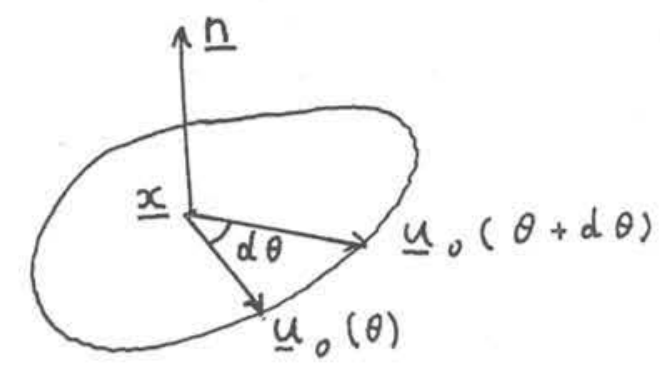

Dans le cas général $u_{0}$ et $1\left(x, u_{0}\right)$ dépendent de $\theta$ et la porosité de surface $\overline{s e}$ déduit $\overline{\text { de }}$ la porosité linéaire par la relation :

$$
f\left(\underline{x}, a_{0} \underline{n}\right)=\frac{\int_{0}^{2 \pi} l\left(\underline{x}, \underline{u}_{0}\right)\left|\underline{u_{0}}\right|^{2} d \theta}{\int_{0}^{2 \pi}\left|\underline{u}_{0}\right|^{2} d \theta}
$$

Dans le cas d'une porosité isotrope $1\left(x, u_{0}\right)$ est indépendant de l'angle $\theta$ et par conséquent :

$$
f\left(\underline{x}, a_{0} \underline{n}\right)=1\left(\underline{x}, \underline{u_{0}}\right)
$$

On définira de même une porosité de volume mais pour cette définition seules interviendront les coordonnées du point considéré :

$$
n(x)=v_{0}^{p} / v_{0}
$$

où le volume $v_{0}$ est défini par la relation :

$$
v_{0}=\int_{0}^{\pi} d \theta \int_{0}^{2 \pi} d \varphi \int_{0}^{\left|u_{0}\right|} \mid \underline{|u|^{2}} \sin \theta \underline{|d u|}
$$

et :

$$
\begin{aligned}
v_{0}^{p}=\int_{0}^{\pi} d \theta & \int_{0}^{2 \pi} d \varphi \\
& \int_{0}^{\left|\underline{u}_{0}\right|} l\left(\underline{x}, \underline{u}_{0}\right) \mid \underline{|u|^{2}} \sin \theta \underline{|d u|}
\end{aligned}
$$

(en coordonnées sphériques $u_{0}$ dépend de $\theta$ et $\varphi$ ).

On observera que si la porosité est isotrope alors :

$$
n(\underline{x})=f\left(\underline{x}, a_{0} \underline{n}\right)=1\left(\underline{x}, \underline{u_{0}}\right)
$$

Le fait que la porosité soit isotrope n'implique pas obligatoirement que $\left|u_{0}\right|$ soit indépendant de l'orientation de $u_{0}$ ( $\theta$ et $\varphi$, dans le cas du système de référence sphérique adopté précédemment) mais seulement que le rapport $\left|u_{0}^{p}\right| /\left|u_{0}\right|$ reste constant dans toutes les directions. La notion d'isotropie pour la porosité fait donc moins intervenir la notion d'orientation préférentielle des pores que celle de distribution préférentielle. Il semble, en première approximation, que pour la plupart des roches, y compris des roches métamorphiques telles que les schistes, on puisse considérer la porosité comme étant isotrope car on peut supposer que dans toutes les directions la distribution des pores est quelconque.

Ayant défini la porosité en un point quelconque d'une masse rocheuse, il est possible de définir un gradient de porosité dans l'espace considéré; celui-ci est défini par la relation suivante :

$$
\nabla l\left(\underline{x}, \underline{u}_{0}\right)=l, \hat{\mathrm{I}}_{i}=\frac{\partial l\left(\underline{x}, \underline{u}_{0}\right)}{\partial x_{i}} \hat{\mathrm{I}}_{i}
$$

où $\hat{I}_{i}$ sont les vecteurs unitaires du repère considéré. Pour les corps à porosité isotrope la relation (11) implique que :

$$
n_{,_{i}}=f_{,_{i}}=1,{ }_{i}
$$

\subsection{Conditions d'équilibre du matériau continu équivalent}

On considère que le liquide s'écoule selon la loi de Darcy et que la porosité est isotrope.

Soit un volume $\mathrm{V}$, à l'intérieur de la masse considérée, de surface $\mathrm{S}$. On appellera $\mathrm{V}_{F}$ la partie de $\mathrm{V}$ occupée par le liquide et $V_{M}$ celle occupée par la matrice solide. On définira de même $\mathrm{S}_{F}$ la partie de $\mathrm{S}$ qui traverse le liquide et $S_{M}$ celle qui traverse le solide. Avec ces notations, l'équilibre dynamique du volume V est représenté par l'équation :

$$
\begin{aligned}
\int_{S_{F}} \underline{\sigma}^{F} \underline{n} d a & +\int_{S_{M}} \stackrel{\sigma}{\sim}^{M} \underline{n} d a+ \\
& +\int_{V_{F}} \rho_{F} \underline{b} d v+\int_{V_{M}} \rho_{M} \underline{b} d v=0
\end{aligned}
$$

où $\sigma^{F}$ et $\sigma^{M}$ sont respectivement les tenseurs des contraintes définis dans le liquide et dans le solide; $\rho_{F}$ et $\rho_{M}$ sont respectivement les densités dans le liquide et dans le solide;

$b$ est la force gravitationnelle (généralement $g \delta_{i 3} \hat{\mathrm{I}}_{i}$ ), $\bar{n}$ est la normale à l'élément de surface $d a=d a n$.

Considérons l'élément d'aire $a_{0}$ de $\mathrm{S}$, ainsi qu'il a été défini pour la porosité de surface. Cet élément est soumis à la force de surface $d t$ dont les composantes 
s'expriment de la façon suivante :

$$
\underline{d t}=\sum_{m} \int_{A_{m}} \sigma_{i j}^{F} n_{j} \hat{I}_{i} d a+\sum_{m^{\prime}} \int_{A_{m^{\prime}}} \sigma_{i j}^{M} n_{j} d a
$$

où l'on suppose que l'aire $a_{0}$ comporte $m$ pores d'aire $\mathrm{A}_{m}$ et $m^{\prime}$ parties solides $\mathrm{A}_{m^{\prime}}$.

Appliquant le théorème de la moyenne, on peut écrire que pour le $m^{\text {isme }}$ pore :

$$
\int_{A_{m}} \sigma_{i j}^{F} n_{j} d a=\left.\sigma_{i j}^{F}\right|_{\underline{y}_{m}} \mathrm{~A}_{m} n_{j}
$$

où $\left.\sigma_{i j}^{F}\right|_{\underline{y}_{m}}$ est la valeur de $\sigma_{i j}^{F}$ au point $\underline{y}_{m}$ de $\mathrm{A}_{m}$.

Il est donc possible de définir la valeur moyenne $\sigma_{i j}^{\prime F}$ de $\sigma_{i j}^{F}$ pour l'aire $a_{0}$ de $\mathrm{S}$ considérée :

$$
\sigma_{i j}^{\prime F}=\left.\sum_{m} \sigma_{i j}^{F}\right|_{y_{m}} n_{j} \mathrm{~A}_{m} / \sum_{m} \mathrm{~A}_{m}
$$

On définira de façon similaire une valeur moyenne des contraintes supportées par la partie solide de l'aire $a_{o}$ :

$$
\sigma_{i j}^{\prime M}=\left.\sum_{m^{\prime}} \sigma_{i j}^{M}\right|_{\underline{y}^{\prime} m^{\prime}} n_{j} \mathrm{~A}_{m^{\prime}} / \sum_{m^{\prime}} \mathrm{A}_{m^{\prime}}
$$

Ainsi la traction supportée par l'aire $a_{o}$ peut s'exprimer par l'une ou l'autre des relations (15) ou (18) :

$$
d t_{i}=f \sigma_{i j}^{\prime F} n_{j} a_{0}+(1-f) \sigma_{i j}^{\prime M} n_{j} a_{0}
$$

Si l'on définit, pour tout point de la roche saturée, la notion de contrainte moyenne par la relation :

$$
\sigma_{i j}=f \sigma_{i j}^{\prime F}+(1-f) \sigma_{i j}^{\prime M}
$$

la relation (18) peut encore s'écrire :

$$
d t_{i}=\int_{a_{0}} \sigma_{i j} n_{j} d a
$$

Dans le modèle proposé, on suppose que lorsque le volume $d v$ de la roche saturée tend vers zéro, il le fait de telle sorte que pour $d \nu<v_{o}\left(v_{o}\right.$ volume défini pour la porosité de volume) la porosité reste constante. Ce modèle n'est donc équivalent à la roche réelle que pour des volumes supérieurs à $v_{0}$. On a, alors, l'équivalence entre l'une ou l'autre des représentations suivantes pour la résultante des forces de surface :

$$
\begin{aligned}
\underline{t}=\int_{S} \underline{d t} & =\int_{S_{F}} \underset{\sim}{\sigma^{F}} \underline{n} d a+\int_{S_{M}} \stackrel{\sigma}{\sim}^{M} \underline{n} d a \\
& =\int_{S} \underset{\sim}{\sigma} \underset{n}{n} d a
\end{aligned}
$$

On peut définir, de même, une densité moyenne en tout point de la roche par la relation :

$$
\rho=f \rho_{F}+(1-f) \rho_{M}
$$

où $\rho_{F}$ et $\rho_{M}$ sont respectivement les densités moyennes du liquide et du solide pour le volume $v_{o}$ (défini pour la porosité de volume).

L'équation (14) peut alors s'écrire :

$$
\int_{S} \tilde{\sigma} \underline{n} d a+\int_{V} \rho \underline{b} d v=0
$$

Ainsi, après avoir transformé l'intégrale de surface en intégrale de volume et après avoir observé que l'équation (15) doit être vérifiée pour tout volume V, on en déduit la relation classique d'équilibre des matériaux continus :

$$
\operatorname{div} \underset{\sim}{\sigma}+\underline{p} \underline{b}=0
$$

qui peut encore s'écrire, si l'on fait intervenir les composantes moyennes des contraintes supportées par le liquide et par le solide défini en tout point du matériau continu équivalent :

$$
\begin{aligned}
f\left(\operatorname{div} \sigma^{\prime F}+\rho_{F}^{\prime} \underline{b}\right)+ & (1-f)\left(\operatorname{div} \underline{\sigma}^{\prime M}+\rho_{M}^{\prime} \underline{b}\right)+ \\
& +\left(\underline{\sim}^{\prime F}-\underline{\sigma}^{\prime M}\right) \operatorname{grad} f=0
\end{aligned}
$$

\section{ELASTICITE LINEAIRE}

\subsection{Théorie}

On supposera ici que la matrice solide ainsi que le matériau global solide + liquide (matériau B) sont linéairement élastiques. On peut alors déterminer aisément la loi de comportement du matériau B en appliquant la décomposition des contraintes indiquée sur la figure 2. La première composante (composante I) correspond à une pression hydrostatique appliquée aussi bien sur la surface extérieure de l'élément considéré qu'à l'intérieur de l'espace poreux interconnecté. Il convient de souligner ici le fait que les pores qui n'appartiennent pas au volume poreux interconnecté sont assimilés à la matrice solide, on définit ainsi un matériau continu $\mathrm{M}$ équivalent à la matrice solide et aux pores non connectés ; la notion de porosité doit alors être comprise comme étant la "porosité interconnectée ».

D'après Nur et Byerlee (1971), on peut montrer que la déformation causée par cette pression hydrostatique s'exprime par la relation :

$$
\varepsilon_{i j}^{I}=\mathrm{P} / \mathrm{K}_{M} \cdot \delta_{i j}
$$

où $1 / K_{M}$ est la compressibilité du matériau équivalent $\mathrm{M}$.
Fig. 2. - Décomposition de l'état de contrainte pour l'analyse de la déformation des roches poreuses et saturées.

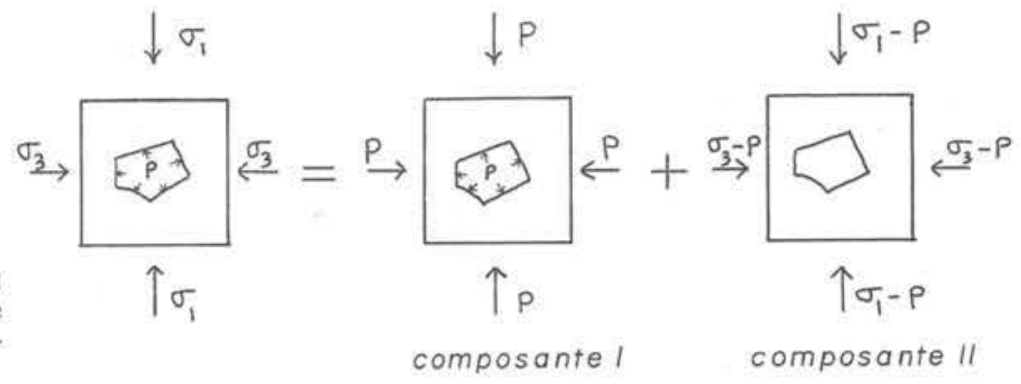


La composante II, que l'on notera $(\sigma-\mathrm{P} 1)$ est appliquée sur le matériau rocheux saturé (matériau B) libre de toute pression interstitielle. La déformation correspondante est donc :

$\varepsilon_{i j}^{I I}=\frac{1+v_{B}}{\mathrm{E}_{B}}\left(\sigma_{i j}-\mathrm{P} \delta_{i j}\right)-\frac{3 v_{B}}{\mathrm{E}_{B}}(\bar{\sigma}-\mathrm{P}) \delta_{i j}$

où $v_{B}$ et $E_{B}$ sont respectivement le coefficient de Poisson et le module d'Young du matériau B.

$\bar{\sigma}=\sigma_{i i} / 3$ est la contrainte sphérique appliquée sur l'élément considéré. La déformation totale est donc :

$\varepsilon_{i j}=\frac{1+\nu_{B}}{\mathrm{E}_{B}} \sigma_{i j}-\frac{3 \nu_{B}}{\mathrm{E}_{B}} \bar{\sigma} \delta_{i j}-\left(\frac{1}{\mathrm{~K}_{B}}-\frac{1}{\mathrm{~K}_{M}}\right) \frac{\mathrm{P}}{3} \delta_{i j}$

où $1 / \mathrm{K}_{B}=3\left(1-2 v_{B}\right) / \mathrm{E}_{B}$ est la compressibilité du matériau $\mathrm{B}$.

On retrouve ainsi aisément le résultat de Geertsma (1957) :

$$
\varepsilon=\varepsilon_{i i}=\frac{\Delta \mathrm{V}_{B}}{\mathrm{~V}_{B}}=\frac{1}{\mathrm{~K}_{B}}(\bar{\sigma}-\mathrm{P})+\frac{\mathrm{P}}{\mathrm{K}_{M}}
$$

où $\varepsilon$ est la déformation volumique.

En appliquant le théorème des travaux réciproques, on obtient le résultat classique (Geertsma 1957) :

$$
\frac{\Delta \mathrm{V}_{P}}{\mathrm{~V}_{P}}=\frac{1}{n}\left(\frac{1}{\mathrm{~K}_{B}}-\frac{1}{\mathrm{~K}_{M}}\right)(\bar{\sigma}-\mathrm{P})+\frac{p}{\mathrm{~K}_{M}}
$$

où $\Delta \mathrm{V}_{P} / \mathrm{V}_{P}$ est la variation relative de volume poreux interconnecté, $n$ est la porosité volumique (elle est égale à la porosité de surface $f$ dans le cas considéré ici).

On en déduit la variation de porosité relative :

$$
\frac{\Delta f}{f}=\left[\frac{1}{f}\left(\frac{1}{\mathrm{~K}_{B}}-\frac{1}{\mathrm{~K}_{M}}\right)-\frac{1}{\mathrm{~K}_{B}}\right](\bar{\sigma}-\rho)
$$

Si la compressibilité $1 / K_{M}$ du matériau $M$ est négligeable devant la compressibilité $1 / \mathrm{K}_{B}$ du matériau $\mathrm{B}$, la relation (28) devient :

$$
\varepsilon_{i j}=\frac{1+v_{B}}{\mathrm{E}_{B}}\left(\sigma_{i j}-\mathrm{P} \delta_{i j}\right)-\frac{3 v_{B}}{\mathrm{E}_{B}}(\bar{\sigma}-\mathrm{P}) \delta_{i j}
$$

Si $1 / K_{M}$ n'est pas négligeable, ce qui est le cas si le volume poreux non interconnecté est important, la variation de pression interstitielle observée pour des conditions non drainées est donnée par :

$$
\Delta \mathrm{P}=\frac{1 / \mathrm{K}_{B}-1 / \mathrm{K}_{M}}{f\left(\mathrm{C}_{F}-1 / \mathrm{K}_{M}\right)+\left(1 / \mathrm{K}_{B}-1 / \mathrm{K}_{M}\right)} \cdot \bar{\sigma}
$$

où $\mathrm{C}_{F}$ est la compressibilité du liquide. Dans ce cas, la déformation du matériau B est donnée par :

$$
\begin{aligned}
\varepsilon_{i j}= & \frac{1+v_{B}}{\mathrm{E}_{B}} \sigma_{i j}-\left[\frac{3 v_{B}}{\mathrm{E}_{B}}-\right. \\
& \left.-\frac{1 / 3\left(1 / \mathrm{K}_{B}-1 / \mathrm{K}_{M}\right)^{2}}{f\left(\mathrm{C}_{F}-1 / \mathrm{K}_{M}\right)+\left(1 / \mathrm{K}_{B}-1 / \mathrm{K}_{M}\right)}\right] \cdot \bar{\sigma}
\end{aligned}
$$

Pour des conditions drainées où l'écoulement obéit à la loi de Darcy, les déplacements dans le matériau B sont calculés en intégrant l'équation (35), obtenue en substituant l'équation (28) dans l'équation d'équilibre (24); en remplaçant $\varepsilon_{i j}$ par $1 / 2\left(u_{i, j}+u_{j, i}\right)$ où $u$ est le vecteur déplacement :

$$
\begin{aligned}
\mathrm{G}_{B} u_{j, i i}+\frac{\mathrm{G}_{B}}{1-2 v_{B}} u_{i, i j}+ \\
\quad+\left(1-\mathrm{K}_{B} / \mathrm{K}_{M}\right) \mathrm{P}_{,_{j}}+\rho b_{j}=0
\end{aligned}
$$

avec $\mathrm{G}_{B}=2\left(1+v_{B}\right) / \mathrm{E}_{B}=$ module de Coulomb du matériau $B$.
Cette équation s'écrit sous forme vectorielle :

$$
\begin{aligned}
\mathrm{G}_{B} \nabla^{2} \underline{u}+ & \frac{\mathrm{G}_{B}}{1-2 v_{B}} \nabla \nabla \cdot \underline{u}+ \\
& +\left(1-\mathrm{K}_{B} / \mathrm{K}_{M}\right) \nabla \mathrm{P}+\rho \underline{b}=0
\end{aligned}
$$

On retrouve ainsi le fait que la force de percolation due au gradient de pression $\nabla \mathrm{P}$ dans le liquide, n'est assimilable à une force de volume que si la compressibilité $1 / K_{M}$ est négligeable devant $1 / K_{B}$.

On remarquera que les équations (24) et (35) sont similaires à celles dérivées par Biot (1941), avec l'équivalence suivante entre les constantes :

$$
\begin{array}{cc}
\text { Biot }(1941) & \text { Dérivation présentée } \\
1 / \mathrm{H} & 1 / \mathrm{K}_{B}-1 / \mathrm{K}_{M} \\
\alpha=2(1+v) \mathrm{G} / & 1-\mathrm{K}_{B} / \mathrm{K}_{M} \\
3 \mathrm{H}(1-2 v) & -1 / \mathrm{K}_{M}\left(\mathrm{~K}_{B} / \mathrm{K}_{M}-1+f\right) \\
\mathrm{l} / \mathrm{Q} &
\end{array}
$$

On observe donc que la constante $\mathrm{Q}$, considérée par Biot, n'est pas vraiment une constante puisque $f$, la porosité isotrope, n'est pas constante.

Dans une dérivation postérieure, Biot (1955) a proposé de différencier la partie de la contrainte, s'exerçant sur un cube représentatif, supportée par la matrice solide de celle qui est supportée par le liquide.

Ainsi que cela a été montré dans le précédent paragraphe, la partie de la contrainte $\sigma$ supportée par la matrice solide peut être représentée par $\sigma^{\prime M}$ et celle supportée par le liquide par $\sigma^{\prime F}=\mathrm{P} 1\left(\mathrm{P}^{\sim}=\right.$ pression interstitielle). Or Biot supposé d'une part que la partie de la contrainte supportée par la matrice $M$ est égale à $(1-f) \sigma^{\prime M}$ et d'autre part que c'est cette contrainte qui est responsable de la déformation $\varepsilon$ décrite par l'équation (28). Il vient d'être montré quẽ la déformation $\varepsilon$ était obtenue si l'on considérait la contrainte totale $\sigma=f \sigma^{\prime F}+(1-f) \sigma^{\prime M}$ aussi l'équation $\left(35^{\prime}\right)$ diffère-t-elle de celle dérivée par Biot en 1955.

On relèvera finalement que l'équation (28) pour $\varepsilon$ est également différente de celle proposée par Lubinsĩi (1954) puisque cet auteur proposait la relation suivante :

$$
\begin{aligned}
\varepsilon_{i j}=\frac{1+v_{B}}{\mathrm{E}_{B}} \sigma_{i j}-\frac{3 \nu_{B}}{\mathrm{E}_{B}} & \bar{\sigma} \delta_{i j}- \\
& -\left(\frac{1-f}{\mathrm{~K}_{B}}-\frac{1}{\mathrm{~K}_{M}}\right) \frac{\mathrm{P}}{3} \delta_{i j}
\end{aligned}
$$

où $f$ est la porosité d'aire supposée constante dans toute la roche.

\subsection{Vérification expérimentale}

Afin de vérifier l'applicabilité de la théorie de l'élasticité linéaire aux roches poreuses et saturées, des éprouvettes cylindriques de deux roches sédimentaires (grès de Béréa et calcaire de l'Indiana), saturées artificiellement par de l'huile légère de faible viscosité, ont été soumises à des efforts de compressions triaxiaux. Au cours du processus de déformation, les déformations axiales et volumiques ainsi que les variations de volume poreux interconnecté ont été mesurées. La technique expérimentale est décrite dans le paragraphe suivant.

Les courbes ainsi obtenues sont représentées sur les figures 5, 6, 7, 8, 9 et 10 . On remarque que la théorie de l'élasticité linéaire fournit des résultats tout a fait satisfaisants pour le calcaire de l'Indiana et que la compressibilité $1 / \mathrm{K}_{M}$ est du même ordre de grandeur, 
bien que plus petite, que le coefficient $1 / K_{B}$ de la roche saturée. Au contraire, pour le grès de Béréa, la définition d'un comportement linéaire à faible pression de confinement apparaît tout à fait subjective alors qu'elle devient plus réaliste dans le domaine des hautes pressions de confinement (supérieur à 200 bars). Pour ce comportement non linéaire un nouveau modèle devrait être défini, ce point est discuté aux paragraphes $4.2,4.3$ et 5.2.

\subsection{La notion de limite élastique}

Lorsque le comportement d'une roche est élastique et linéaire, la limite élastique est définie comme étant l'effort maximal pour lequel la relation effort-déformation reste linéaire. Toutefois, ce point est parfois difficile à définir et il est connu, par exemple, que la limite de linéarité pour la relation effort-déformation volumique est généralement plus faible que celle définie à partir de la relation effort-déformation axiale (Brace et al. (1966), Morlier (1969). De plus, cette limite de linéarité n'est évidemment pas définie pour les matériaux non linéaires bien qu'élastiques. Nous proposons donc de définir cette limite par comparaison entre le travail des forces extérieures appliquées à l'éprouvette et l'énergie élastique supposée être accumulée dans la roche pour une déformation donnée.

$\mathrm{Si}$ le travail des forces extérieures est supérieur ou égal à l'énergie de déformation élastique calculée, on peut considérer que la roche est encore dans le domaine élastique et que la différence entre le travail des forces extérieures et l'énergie de déformation élastique correspond à la quantité d'énergie absorbée par les phénomènes de frottement. Au contraire, lorsque le travail des

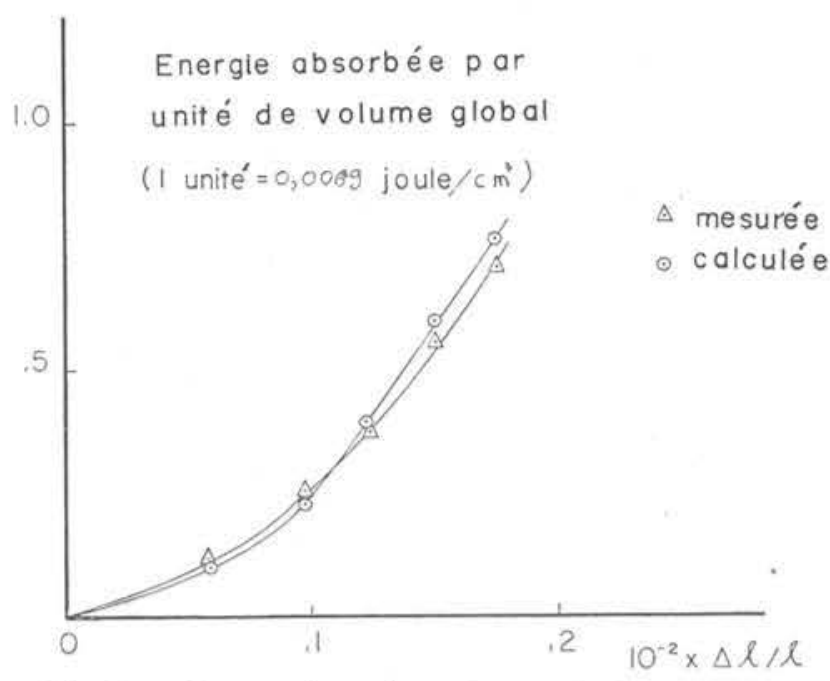

Fig. 3. - Comparaison des valeurs calculées et mesurées pour l'énergie de déformation accumulée dans la matrice solide par unité de volume global d'un échantillon saturé de calcaire de l'Indiana.

forces extérieures devient inférieur à l'énergie de déformation élastique, cela indique qu'un nouveau phénomène est intervenu dans le mode de déformation, à savoir rupture fragile ou déformation plastique, et que, par conséquent, les conditions de chargement n'appartiennent plus au domaine de définition du comportement élastique. Les figures 3 et 12 indiquent qu'une telle définition devrait apporter des résultats satisfaisants.

\section{ETUDE DE LA DESINTEGRATION QUASISTATIQUE SOUS CONTRAINTE TRIAXIALE DE COMPRESSION EN CONDITIONS DRAINEES}

\subsection{Etude expérimentale}

Afin d'analyser l'influence mécanique d'un liquide sur le processus de désintégration d'une roche saturée, il est apparu nécessaire d'individualiser tout d'abord les différents mécanismes mis en jeu.

Une série d'essais triaxiaux drainés, effectués sur des éprouvettes cylindriques de grès de Béréa et de calcaire de l'Indiana (diamètre $5 \mathrm{~cm}$, élancement 2) au moyen d'une presse servo-asservie, ont permis d'obtenir des informations sur le comportement de ces deux roches après que leur limite élastique ait été dépassée et avant que la résistance résiduelle ne soit atteinte (domaine appelé ici domaine post-élastique).

Ces deux roches ont été choisies car elles présentent toutes deux une forte porosité ( $18 \%$ pour le grès, $15 \%$ pour le calcaire) mais des perméabilités très différentes (perméabilité à l'eau pour le grès de $0.58 \mu \mathrm{m} / \mathrm{s}$, pour le calcaire de $0.007 \mu \mathrm{m} / \mathrm{s}$ ) ainsi que des comportements rhéologiques complémentaires (fragile pour le grès, tendance ductile pour le calcaire quand la pression de confinement devient supérieure à 175 bars).

Ces essais ont permis de compléter certains résultats publiés précédemment sur ce sujet (par exemple Robinson (1959), Handin et al. (1963), Baron et al. (1963), Brace et al. (1966), Brace and Martin (1968), Wawersik (1968), Edmund and Paterson (1971), Schok et al. (1973)). Ils seront discutés après que la technique expérimentale ait été décrite.

\subsubsection{Technique expérimentale}

Les essais triaxiaux drainés, effectués sur des éprouvettes saturées artificiellement mais indépendamment des essais proprement dits, ont été réalisés au moyen d'une presse asservie de façon à assurer continuellement un mode de déformation quasistatique.

Le principe des presses servo-asservies a déjà été exposé (Rummel and Fairhust (1970), Hudson et al. (1971)) et nous nous contenterons de décrire la technique expérimentale ainsi qu'elle est schématisée sur la figure 4. La première boucle d'asservissement impose que la pression de confinement soit maintenue constante tout au long de l'essai. Ainsi, si la pression a tendance à diminuer, la charge axiale est augmentée, ce qui fait pénétrer le piston de chargement à l'intérieur de la cellule et donc augmente la pression de confinement. Au contraire, si la pression de confinement a tendance à augmenter, la charge axiale est diminuée, ce qui se traduit par une sortie du piston et donc une diminution de pression de confinement, Le deuxième circuit d'asservissement impose un écoulement du fluide de confinement monotone avec le temps. Ce circuit impose donc une diminution de pression à l'intérieur de la cellule et, par réaction de la première boucle, un chargement de l'éprouvette tel que sa déformation latérale moyenne $\left(\varepsilon_{22}+\varepsilon_{33}\right)$ soit une fonction monotone du temps. 


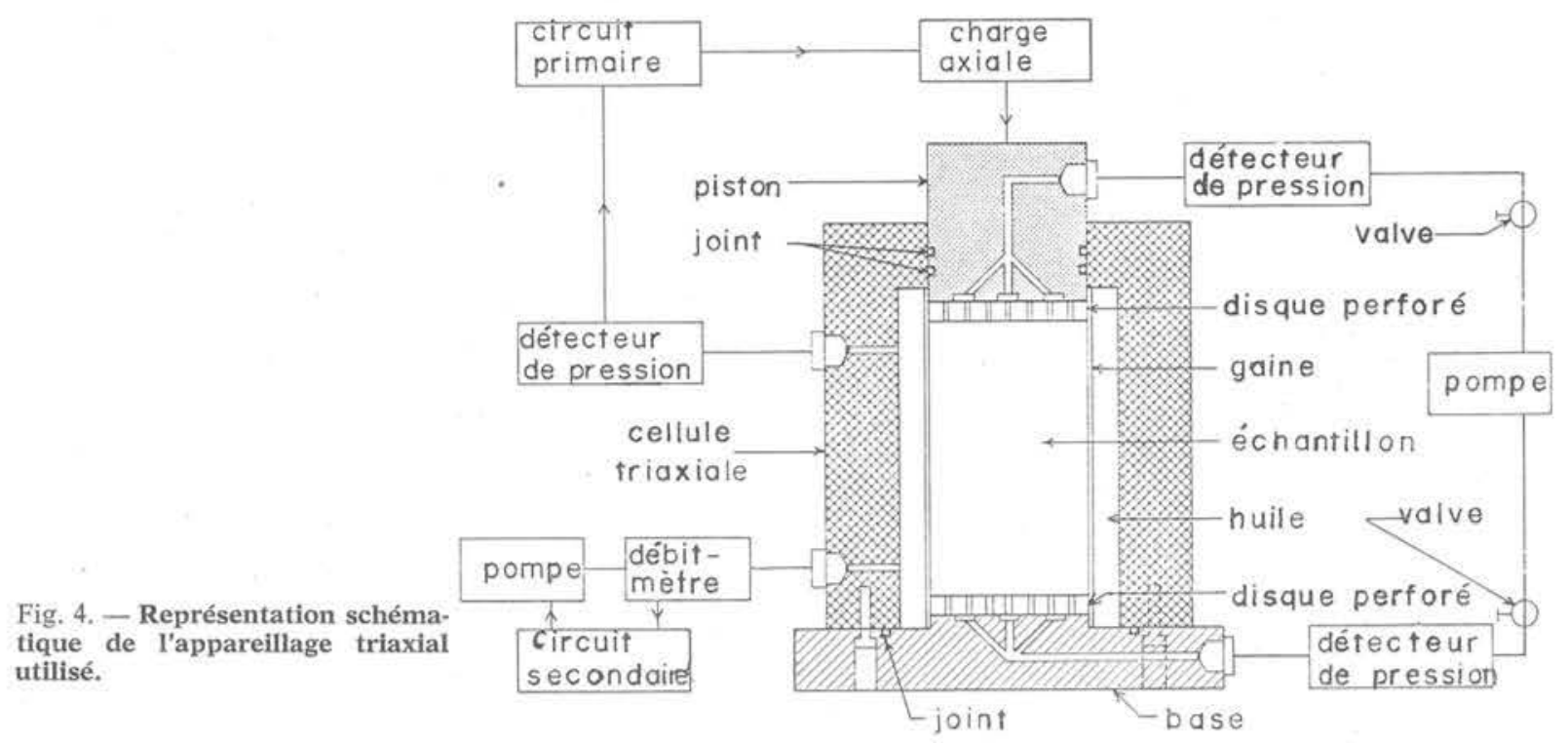

La mesure de la quantité de fluide s'écoulant hors de la cellule triaxiale ainsi que celle des déplacements du piston permettent de déterminer la déformation axiale moyenne et la déformation volumique moyenne de l'éprouvette saturée (c'est-à-dire du matériau B défini dans le paragraphe précédent). Les déformations ont lieu de façon quasistatique (vitesse de déformation latérale moyenne de $10^{-6} \mathrm{~s}^{-1}$ ) ce qui permet d'assurer manuellement la constance de la pression interstitielle tout au long de l'essai. Les quantités de liquide s'écoulant hors, ou vers, l'éprouvette fournissent une mesure directe de la variation de volume poreux interconnecté.

\subsubsection{Résultats}

Les figures 5 à 10 fournissent une synthèse des résultats obtenus. Il est à noter que ces courbes ne correspondent pas aux courbes effort-déformation de la rhéologie mais à des courbes force moyenne par unité de surface-déformation moyenne par unité de longueur, surface ou volume. En effet, si dans le domaine élastique, on peut, en première approximation, supposer que la relation effort-déformation peut être étudiée par des essais triaxiaux, au cours de la désintégration de l'éprouvette la présence de nombreuses discontinuités rend illusoire la détermination exacte des contraintes à l'intérieur de l'éprouvette à partir de la seule connaissance des forces appliquées. Ainsi, pour chaque essai, a-t-on déterminé les trois courbes suivantes :

- Courbe a : force axiale moyenne-déplacement axial moyen $(\Delta 1 / 1)$;

- Courbe b : force axiale moyenne-variation moyenne du volume global $\left(\Delta \mathrm{V}_{B} / \mathrm{V}_{B}\right)$;

- Courbe c : force axiale moyenne-variation moyenne du volume poreux interconnecté par unité de volume global $\left(\Delta \mathrm{V}_{p} / \mathrm{V}_{B}\right)$.

Les figures $5,8,9$ montrent clairement le phénomène de dilatance classiquement décrit dans la littérature. On observe cependant que, pour ces deux roches, la variation de volume poreux interconnecté et les variations de volume globales de l'échantillon sont similaires mais non identiques. Ainsi, pour la première série d'essais sur le grès (fig. 5) les variations de volume poreux interconnecté sont toujours restées plus faibles que les variations de volume globale alors que le contraire a été observé pour les autres essais. Etant donné qu'aucun gradient de pression interstitielle ne s'est développé (lorsque l'éprouvette était maintenue sous charge constante après une période de chargement, aucune variation de pression de pore n'était observée), on peut en déduire que dans le premier cas un certain nombre de microfissures se sont développées, ou se sont ouvertes, mais sont restées sèches alors que dans le second cas, des pores qui étaient isolés du système interconnecté se sont trouvés reliés à lui par le développement de fissures. Ce dernier effet dépend d'ailleurs de la pression de confinement, étant donné qu'elle influence le nombre de pores isolés du réseau interconnecté.

D'autre part, les résultats obtenus pour le grès indiquent clairement qu'une même roche peut présenter un mode de rupture stable ou instable selon l'amplitude de la pression de confinement et que, contrairement à ce qui est généralement admis, une augmentation de pression n'est pas automatiquement accompagnée d'une plus grande stabilité.

Rappelons la classification proposée par Wawersik (1968) pour caractériser le comportement post-élastique des roches à savoir la classe I lorsqu'il faut fournir continuellement de l'énergie à la roche pour la déformer et la classe II lorsque le développement quasistatique de la rupture requiert qu'une certaine quantité d'énergie soit soustraite (par déchargement des plateaux de la presse) à l'énergie de déformation élastique accumulée dans le roche (cette classification introduit, en fait, la notion de stabilité vis-à-vis du travail des forces extérieures plutôt que vis-à-vis des forces extérieures ellesmêmes).

On observe ainsi que, pour le grès, lorsque son comportement est de classe II, l'augmentation de volume produit par le développement de la rupture reste plus petite que la diminution causée par le relâchement des charges appliquées, effet qui donne naissance à une certaine "contractance ». Cette " contractance » reflète en fait un comportement non linéaire élastique marqué ; il est causé par la présence des nombreuses microfissures développées avant la formation de la surface de macro-rupture.

Plus la densité de microfissures au moment du déclenchement de la rupture instable sera importante et plus grande sera l'instabilité car plus petite étant la raideur de la roche plus grande sera l'énergie élastique 


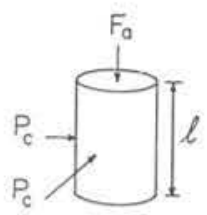

$P_{c}=$ constante $F_{\mathrm{a}}=$ variable
$P_{C}=68 b$

$P_{p}=34 b$

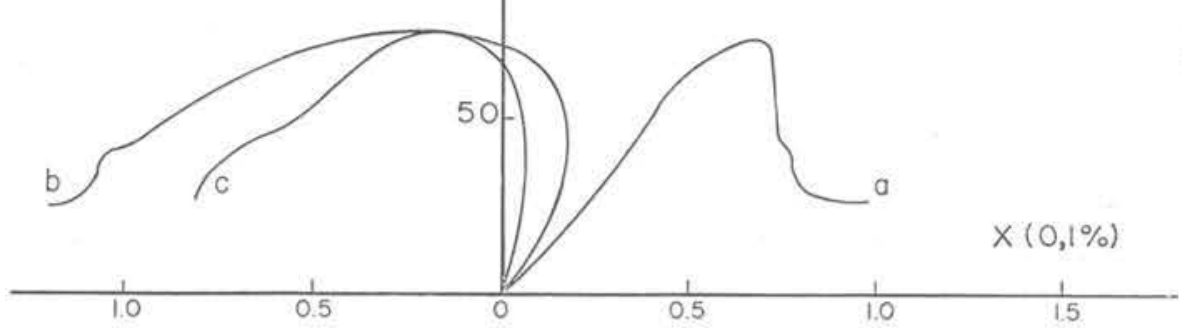

Fig. 5.

Fig. 5 à 7 . - Essais triaxiaux drainés sur le grès de Berea : axe des $\mathrm{x}$ :

- courbe a, déformation axiale moyenne $\Delta 1 / 1$;

- courbe b, déformation volumique globale moyenne $\Delta \mathrm{V}_{\mathrm{B}} / \mathrm{V}_{\mathrm{B}}$;

- courbe c, variation de volume poreux interconnecté par unité de volume global; $\Delta \mathrm{V}_{\mathrm{p}} / \mathrm{V}_{\mathrm{B}}$.
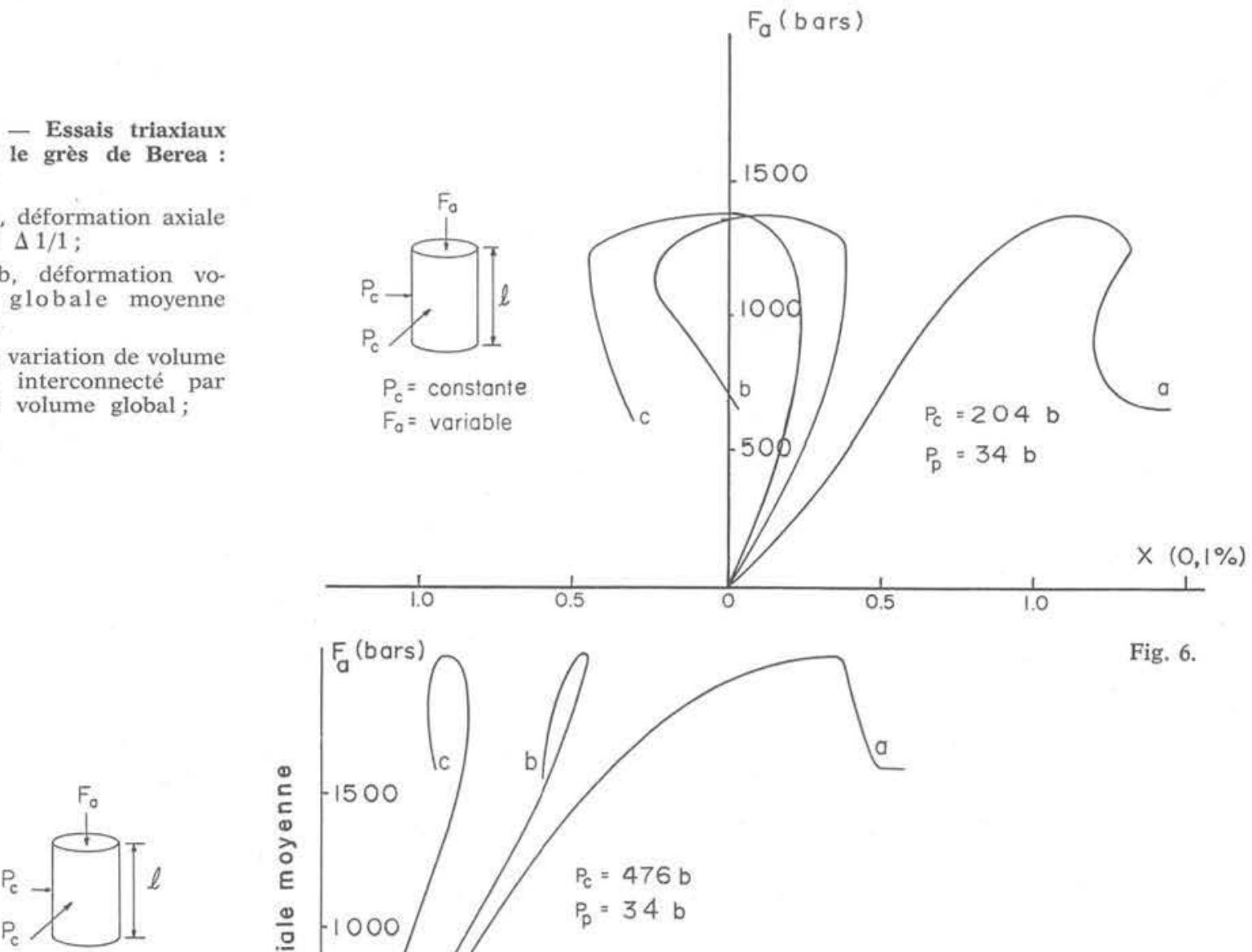

$\mathrm{P}_{\mathrm{c}}=$ constante $F_{0}=$ variable

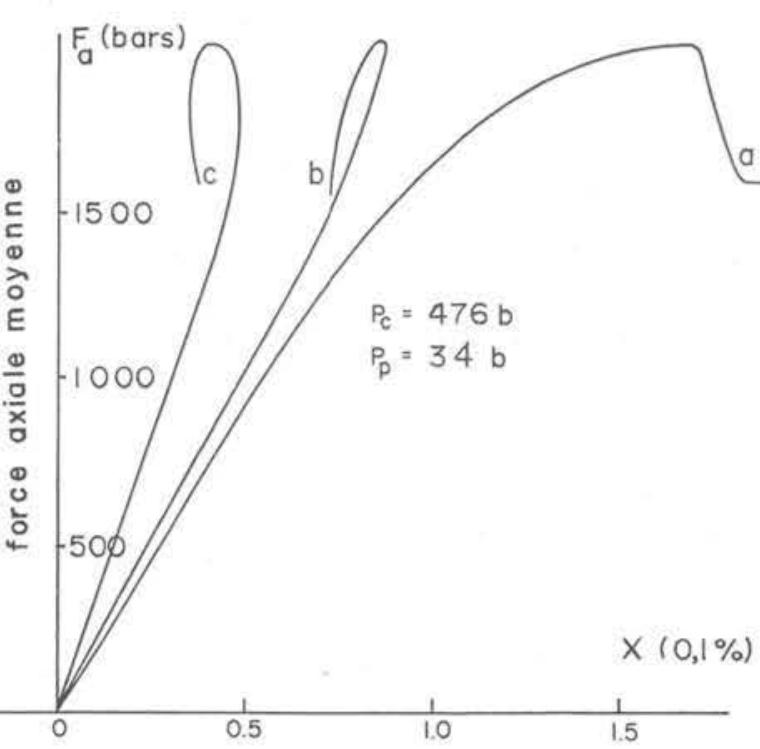

Fig. 7.

susceptible d'être libérée. C'est ce phénomène que reflète indirectement l'effet de "contractance » précédemment décrit.

Notons finalement le deuxième type de «contrac- tance », observé pour le calcaire, et qui correspond à un comportement de type ductile. Cette " contractance » n'est pas un phénomène élastique ; il correspond à ce que les géologues appellent l'écoulement cataclastique 


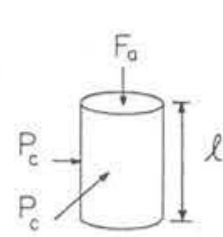

Fig. 8 .

Fig. 8 à 10. - Essais triaxiaux drainés sur le calcaire de l'Indiana :

axe des $\mathrm{x}$ :

- courbe a, déformation axiale moyenne $\Delta 1 / 1$;

- courbe b, déformation volumique globale moyenne $\Delta \mathrm{V}_{\mathrm{B}} / \mathrm{V}_{\mathrm{B}}$

- courbe c, variation de volume poreux interconnecté par unité de volume global ; $\Delta \mathrm{V}_{\mathrm{P}} / \mathrm{V}_{\mathrm{B}}$.

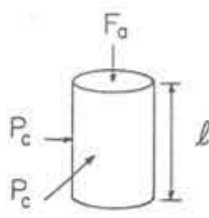

$\mathrm{P}_{\mathrm{c}}=$ constante $\mathrm{F}_{\mathrm{a}}=$ variable

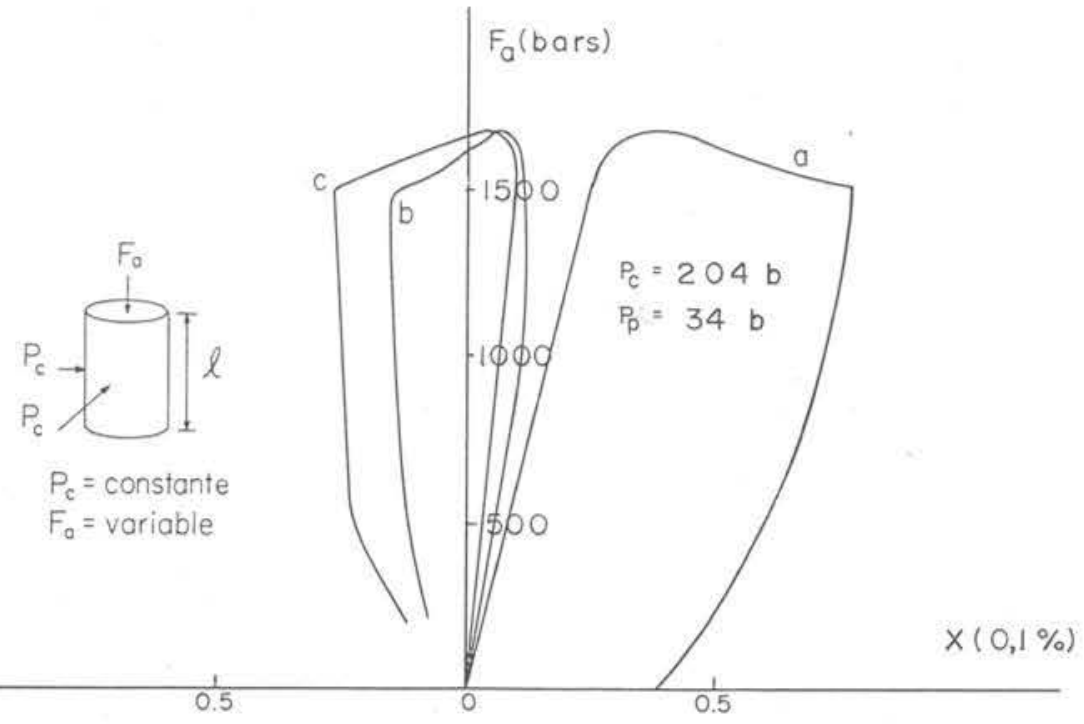

Fig. 9.

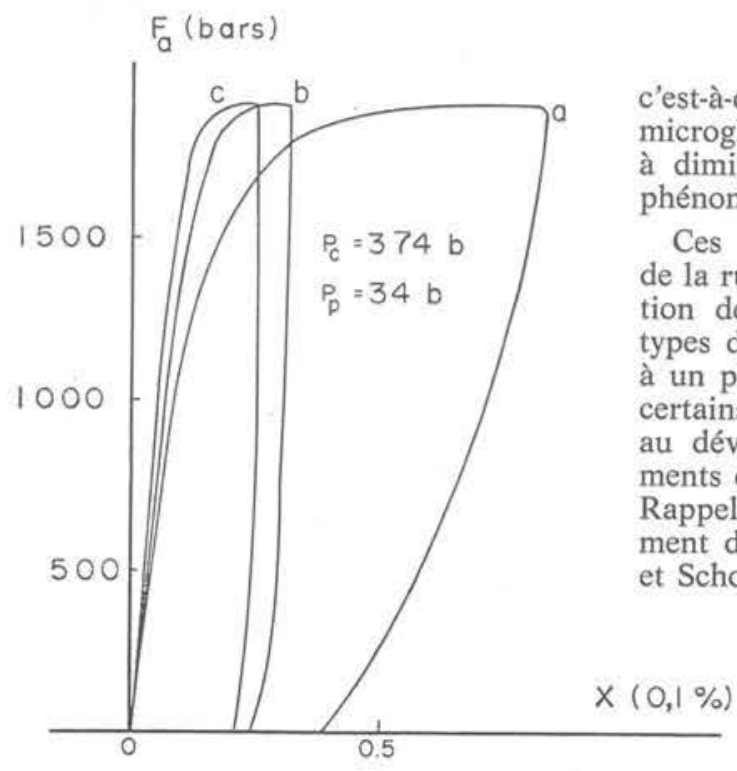

Fig. 10. 


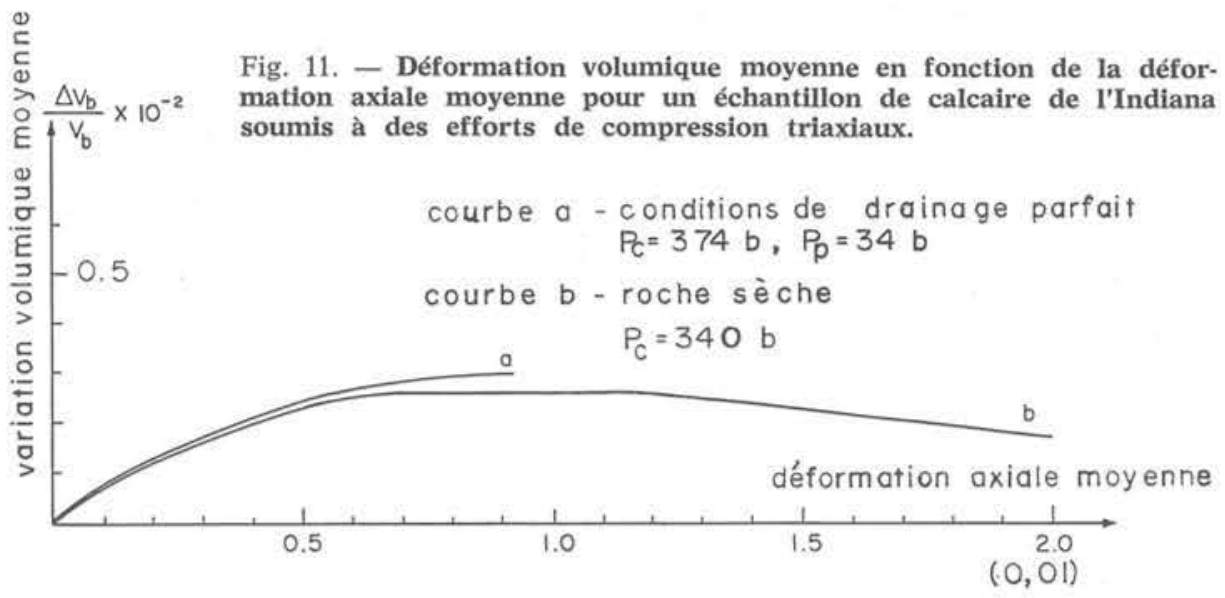

\subsection{Essais d'analyse}

\subsubsection{Fissures et microfissures}

Il est maintenant admis que des discontinuités dues à des ruptures locales se développent dans une roche lorsque celle-ci est soumise à des efforts supérieurs à sa limite élastique. Il est toutefois nécessaire de différencier deux types de discontinuités : les microfissures et les fissures.

Les microfissures ont des dimensions du même ordre de grandeur que celles des éléments constitutifs de la roche. Elles sont à l'origine des variations de volume global observées dans le domaine post-élastique. On essaiera de représenter leur développement et leur influence sur le comportement de la roche en imposant certaines propriétés particulières au matériau continu équivalent $\mathrm{B}$.

Les fissures sont définies de façon macroscopiques ; elles sont la cause de la rupture totale des éprouvettes. Elles peuvent être issues soit de la coalescence de nombreuses microfissures soit de l'extention d'une seule. On étudiera leur développement et leur influence sur le comportement de la roche en les assimilant à des discontinuités dans le champs des déplacements.

\subsubsection{Dilatance et « contractance »}

Le terme de dilatance est généralement utilisé pour décrire l'augmentation de volume associée à l'application d'une contrainte de cisaillement pur. Aussi, plutôt que d'employer les termes dilatance et "contractance », il parait plus simple de parler de dilatance positive pour les augmentations de volume global et de dilatance négative pour les diminutions en conservant le terme dilatance, sans préciser si elle est positive ou négative, pour représenter les variations de volume non prévisibles par la théorie de l'élasticité linéaire.

Etant donné cette définition, deux types de dilatance doivent être distingués :

- l'un, réversible, fait intervenir le comportement élastique non linéaire d'une roche;

- l'autre, irréversible, fait intervenir le développement de microfissures et est associé au processus de désintégration.

\section{Dilatance réversible}

Il a été proposé (Stuart and Dietrich (1974); Freudenthal (1975)) d'associer la dilatance réversible à un comportement élastique non linéaire isotrope en faisant appel au théorème de représentation des fonctions tensorielles isotropes (Truesdell (1952)). Toutefois, cette proposition est très simplificatrice car elle néglige les phénomènes de frottement. Des expériences simples de charge et décharge d'échantillons microfissurés indiquent que la quantité d'énergie dissipée par les frottements est d'autant plus élevée que la densité de microfissures est plus grande et que la variation de contrainte au cours du cycle de chargement est plus importante.

En outre, le théorème proposé par Truesdell ne s'applique, par définition, qu'à des fonctions tensorielles isotropes ; or, la déformation des roches fissurées n'est pas toujours une fonction isotrope de la contrainte. En effet, les microfissures sont souvent orientées dans une direction priviligée (celle de la contrainte principale maximale existant lors du développement des microfissures) ce qui entraîne un comportement anisotrope du matériau continu équivalent $\mathrm{B}$. Ce point a été récemment confirmé par les travaux de Hadley (1975) sur l'anisotropie des vitesses de propagation des ultrasons dans les roches microfissurées.

Toutefois cette représentation, même simpliste, du comportement non linéaire élastique mérite d'être retenue car elle fournit le seul élément de calcul actuellement disponible pour représenter la dilatance réversible.

Rappelons que le théorème de la représentation des fonctions isotropes permet d'écrire la relation effortdéformation sous la forme :

$$
\varepsilon=\varnothing_{0} \underset{\sim}{1}+\varnothing_{1} \underset{\sim}{\sigma}+\varnothing_{2} \underset{\sim}{\sigma^{2}}
$$

où $\underset{\sim}{\varepsilon}=\frac{1}{2}\left(\nabla \underline{u}+\nabla \underline{u}^{T}\right)$ est le tenseur déformation;

$u$ est le vecteur déplacement,

$\bar{\sigma}$ est le tenseur contrainte,

$\tilde{\varnothing}_{k}$ sont des fonctions polynomiales des invariants du premier ordre de $\sigma$.

Si l'on ne retient que les termes d'ordre inférieur ou égal à deux et que l'on prend comme état de référence l'état indéformé, l'équation devient :

$$
\begin{aligned}
\varepsilon_{i j}=\left(a \mathrm{I}_{1}+b \mathrm{I}_{1}^{2}\right. & \left.+c \mathrm{I}_{2}\right) \delta_{i j}+ \\
& +\left(d+e \mathrm{I}_{1}\right) \sigma_{i j}+g \sigma_{i k} \sigma_{k j}
\end{aligned}
$$

avec :

$$
\mathrm{I}_{1}=\sigma_{i i}=\operatorname{tr} \underline{\sigma}
$$




$$
\begin{aligned}
& \mathrm{I}_{2}=\frac{1}{2} \sigma_{i i} \sigma_{j j}(i \neq j)=\frac{1}{2}\left[t r{\underset{\sim}{\sigma}}^{2}-(t r \underset{\sim}{\sigma})^{2}\right] \\
& \mathrm{I}_{3}=\pi \sigma_{i i}=\operatorname{det} \underset{\sim}{\sigma}
\end{aligned}
$$

Pour un tel comportement, six constantes doivent être déterminées, ce qui nécessite que des essais polyaxiaux (triaxiaux vrais) soient effectués.

Les coefficients $a$ et $d$ sont, en fait, les constantes classiques de l'élasticité linéaire plus souvent notées $-\frac{v}{E}$ et $\frac{1+v}{E}$ où $v$ est le coefficient de Poisson et E le module d'Young.

\section{Dilatance irréversible}

Comme cela a déjà été dit, la dilatance irréversible est due au développement de microfissures. Toutefois, bien que l'on puisse essayer de corréler directement la dilatance à la croissance des microfissures, il semble qu'une approche globale, où la roche est assimilée à un matériau continu soit plus prometteuse. C'est ce qu'ont proposé Rudniki et Rice (1975) et Rice (1975). Toutefois, leur modèle, bien qu'il permette de préciser et formaliser mathématiquement certaines caractéristiques du comportement post-élastique des roches, implique que le facteur de dilatance $\beta=d^{P} \varepsilon / d^{P} \gamma$ (où $d^{P} \varepsilon$ est la variation volumique irréversible et $d^{P} \gamma$ est la déformation de cisaillement irréversible) soit déterminé au laboratoire pour différentes conditions de contraintes et de déformation puisque $\beta$ n'est pas constant. Ce modèle représente donc plus une analyse des conséquences qu'une étude du développement proprement dit de la dilatance. Le problème de la modélisation du développement de la dilatance irréversible reste donc à résoudre.

\subsubsection{Le développement des fissures et la notion de résistance}

Le terme fissure doit être compris ici comme représentant des discontinuités macroscopiques dans le champ des déplacements définis dans le matériau continu équivalent $\mathrm{B}$.

Pour des conditions quasistatiques, la propagation de ces fissures peut être analysée en considérant une généralisation du critère de Griffith (Griffith (1920)) à savoir qu'une fissure se propage si la quantité d'énergie élastique libérée par cette propagation est supérieure à celle absorbée par le développement de nouvelles surfaces. Ce critère, qui est dérivé du théorème de l'énergie potentielle minimale, est représenté par la relation

$$
\Delta \mathrm{W}_{E}(\underline{d s}) \geqslant \Delta \mathrm{W}_{B}(\underline{d s})
$$

où $\Delta \mathrm{W}_{E}(d s)$ est la variation d'énergie élastique impliquée par le développement de $d s$;

$\Delta \mathrm{W}_{B}(d s)$ est la quantité d'énergie absorbée par la formation de $\underline{d s} ; \underline{d s}=\underline{n}$ da est la surface nouvellement créée.

Griffith a supposé de plus que $\Delta \mathrm{W}_{B}(d s)$ était directement proportionnel à l'aire des surfaces créées :

$$
\Delta \mathrm{W}_{B}(\underline{d s})=\gamma d a
$$

où $\gamma$ est l'énergie de surface du matériau considéré; $d a$ est l'aire de la surface $d s$.

Le critère de Griffith s'exprime donc par la relation :

$$
\Delta \mathrm{W}_{E}(\underline{d s}) \geqslant \gamma d a
$$

ou encore :

$$
\frac{\partial \mathrm{W}_{E} \underline{(d s)}}{\partial a} \geqslant \gamma
$$

La relation (39) apparaît donc comme plus générale que l'équation (41') puisqu'elle fait intervenir la notion d'orientation de la surface $d s$ considérée. Pour un matériau homogène, l'influence de l'orientation de $d s$ ne fait que refléter l'anisotropie éventuelle du matériau. Pour un matériau hétérogène, tel qu'une roche, il permet d'inclure l'effet possible du gradient des contraintes en tête de fissure. En effet, ainsi que l'ont montré Hoagland et al. (1972), lorsqu'une fissure de traction se développe dans une roche, une zone d'intense microfissuration se forme en tête de fissure et absorbe donc une quantité non négligeable d'énergie. L'étendue de cette zone dépend de la distribution des contraintes en fond de fissure ou plus précisément du gradient de contrainte. Aussi, pour les roches, l'influence de l'orientation de $d s$ fait-elle intervenir l'anisotropie possible ainsi que cet effet du gradient de contrainte. En fait, dans les calculs actuels on suppose encore que le critère énergétique de Griffith reste valide dans sa forme initiale. Un modèle numérique à deux dimensions (conditions de déformations planes), développé à partir du critère exprimé par l'équation (41') et utilisant la méthode des discontinuités de déplacement proposé par Crouch (1975), permet en effet d'obtenir une modélisation satisfaisante de l'influence de la contrainte principale minimale sur l'orientation de la propagation des fissures soumises à des contraintes de compression (Cornet 1976).

Pour des conditions données de vitesse de chargement, de géométrie d'échantillon et de conditions aux frontières, on devrait théoriquement pouvoir établir une relation entre énergie de surface et résistance à la compression puisque la notion de résistance ne fait que refléter la notion de stabilité, ou plutôt d'instabilité, des fissures vis-à-vis des forces apliquées. Cependant, étant donné que l'on ne sait pas encore modéliser correctement le développement de la microfissuration, cette relation n'a encore pu être dérivée.

\subsection{Influence de la pression interstitielle}

L'essai d'analyse du développement de la désintégration des roches a montré qu'essentiellement trois points devaient être considérés :

- formation des microfissures ou développement de la dilatance irréversible ;

- comportement élastique de la roche microfissurée ;

- initiation et propagation des fissures ; étude de leur stabilité vis-à-vis des forces appliquées (notion de résistance), vis-à-vis du travail des forces appliquées (notions de classe I classe II).

Si l'espace des pores est quasiment complétement interconnecté, en sorte que les fissures et microfissures sont presque toutes saturées par le liquide interstitiel, la compressibilité du matériau $M$, équivalant à la matrice solide et aux pores non interconnectés, peut être considérée constante et donc indépendante de l'état de déformation du matériau B. Dans ces conditions, on peut déduire le comportement de la roche microfissurée par superposition des états de contrainte indiqués sur la figure 2 (en fait d'ailleurs, dans ces conditions, la 
déformation dûe à la pression $\mathrm{P} 1$ est souvent négligeable devant celle causée par l̃'état de contrainte $\sigma-\mathrm{P} 1)$. Si le comportement de la matrice $\mathrm{M}$ n'est pas linéaire, le principe de superposition n'est plus applicable et une autre approche doit être proposée. La théorie des milieux continus inter-actifs (Green et Steel 1966) devrait fournir la solution. Il n'est pas certain cependant que l'ampleur du phénomène justifie la complexité d'une telle démarche et le principe de superposition, même s'il est légèrement erroné, devrait fournir des résultats satisfaisants dans la plupart des cas.

Reste donc à résoudre le problème de l'influence de la pression interstitielle sur les ruptures fragiles (fissures et microfissures), c'est-à-dire sur les variations d'énergie de déformation élastique dans la matrice solide, si I'on adopte le critère de Griffith, car seul le solide peut se rompre.

Considérons tout d'abord l'essai triaxial classique et analysons ce qui se passe dans la matrice solide lorsque la charge axiale est augmentée de $\Delta \sigma_{1}$ tandis que la pression de confinement $P_{C}$ et la pression interstitielle $\mathrm{P}_{p}$ sont maintenues constantes. La différence entre le travail des forces extérieures et le travail effectué par la pression de pore fournit le travail effectué sur le matériau continu équivalent $\mathrm{M}$. Si l'on suppose ce matériau $\mathrm{M}$ ainsi que le matériau $\mathrm{B}$ être linéairement élastiques, en appliquant les résultats du paragraphe 3.1, on montre que la variation d'énergie élastique $\Delta \mathrm{W}_{M}$ dans le matériau $M$ associé à la variation $\Delta \sigma_{1}$ est :

$$
\begin{aligned}
\Delta \mathrm{W}_{M}=\frac{1}{2} \Delta \sigma_{1} \Delta \varepsilon_{1} & +\mathrm{P}_{C} \cdot \frac{\Delta \sigma_{1}}{3} \cdot \frac{1}{\mathrm{~K}_{B}}- \\
& -\mathrm{P}_{P} \cdot \frac{\Delta \sigma_{1}}{3} \cdot\left(\frac{1}{\mathrm{~K}_{B}}-\frac{1}{\mathrm{~K}_{M}}\right)
\end{aligned}
$$

où $\Delta \varepsilon_{1}=\left(\frac{\Delta \sigma_{1}}{E_{B}}\right)$ est la déformation axiale du matériau $\mathrm{B}$.

Ainsi, si $1 / K_{M}$ est négligeable devant $1 / K_{B}$, le travail effectué sur $M$, et donc sur la matrice solide si l'on suppose vides les pores non connectés, est le même que si la pression de pore était nulle et la pression de confinement égale à $\mathrm{P}_{C}-\mathrm{P}_{p}$.

En généralisant ce résultat, on montre que pour les matériaux linéairement élastiques et fragiles la variation d'énergie de déformation élastique dans la matrice dépend des variations de la contrainte $(\sigma-\mathrm{P} 1)$ où $\sigma$ et $\mathrm{P}$ sont définis pour tout point du matériau continu équivalent, si $1 / \mathrm{K}_{M}$ est négligeable devant $1 / \mathrm{K}_{B}$. On en déduit donc que pour de tels matériaux les conditions requises pour le développement de ruptures fragiles ne dépendent que de $(\underset{\sim}{\sigma}-\mathrm{P} \underset{\sim}{1})$.

Or ce résultat a également été observé pour le grès de Béréa, matériau qui a été décrit comme étant fortement non linéaire; la notion de linéarité ne devrait donc pas intervenir. Considérons le raisonnement physique suivant.

Les ruptures fragiles (fissures et microfissures) sont dues à des instabilités énergétiques causées par des concentrations de contrainte locales. Si l'espace des pores est entièrement interconnecté et si la matrice solide est homogène, la composante hydrostatique de la décomposition de contrainte indiquée sur la figure 2 induit simplement une contrainte hydrostatique dans le solide et les ruptures ne peuvent donc être causées que par la composante $(\sigma-\mathrm{P} 1)$. Toutefois, si les pores ne sont pas tous interconnectés, ou si la matrice solide est très hétérogène, la composante hydrostatique $\mathrm{P} 1$ peut donner naissance à de fortes concentrations dẽ contraintes et le principe précédent ne sera plus valide.

La figure 12 représente les variations de travail effectué sur le matériau $\mathrm{M}$ en fonction de la déformation axiale moyenne pour des échantillons de calcaire de l'Indiana soumis à des pressions de confinement $\mathrm{P}_{C}$ et des pressions interstitielles $P_{P}$ différentes mais telles que leur différence $\mathrm{P}_{C}-\mathrm{P}_{P}$ soit la même pour tous les essais. On observe que pour des valeurs de $P_{P}$ supérieures à 175 bars le principe selon lequel le travail effectué sur le matériau $M$ ne dépend que de ( $\sigma-\mathrm{P} 1)$ ne s'applique plus. Pour cette roche, le volume poreux non interconnecté est important et il est permis de penser que la valeur 175 bars correspond à la valeur de la pression interstitielle pour laquelle les concentrations de contraintes qu'elle induit ne sont plus négligeables.

On ajoutera d'ailleurs que si la pression de pore est trop élevée, le comportement rhéologique du matériau $\mathrm{M}$ est modifié et qu'en conséquence, la théorie précédemment proposée ne s'appliquerait pas.

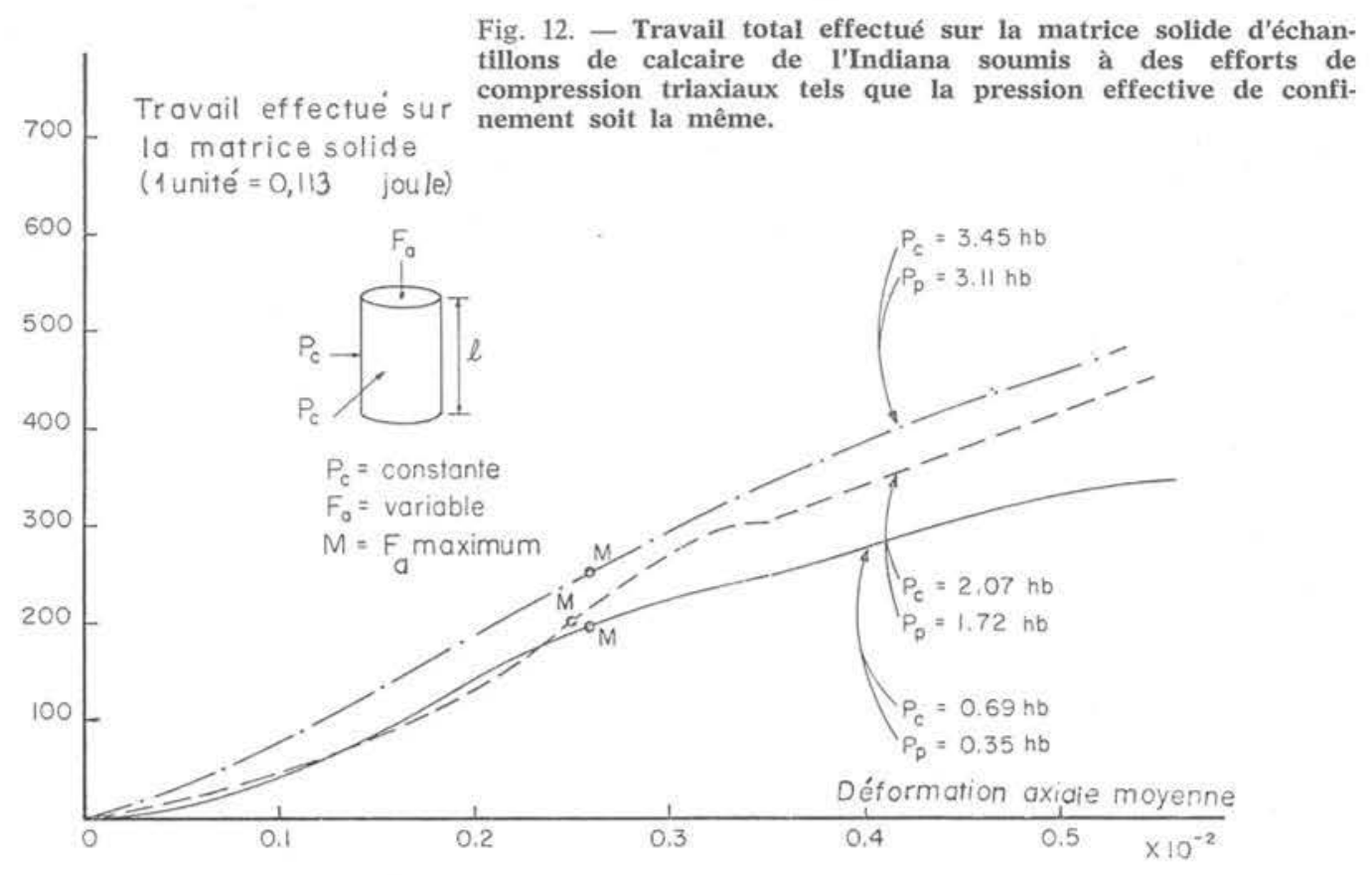




\section{APPLICATION AUX ROCHES NON DRAINEES ET A DRAINAGE PARTIEL}

\subsection{La notion de contrainte effective}

Il est connu depuis longtemps que le comportement des roches saturées est influencé par la pression interstitielle (voir par exemple Terzaghi (1945), Robinson (1959), Baron et al. (1963), Handin et al. (1963), Brace and Martin (1968)). Il a été proposé, en conséquence, d'analyser le comportement des roches saturées de la même façon que celui des roches sèches en remplaçant toutefois la notion de contrainte par celle de contrainte effective. Trois définitions ont été proposées pour ces contraintes effectives et une certaine confusion en est résultée. Ces trois conceptes seront analysés maintenant en utilisant les résultats précédemment décrits.

\subsubsection{Définition classique de la notion de contrainte effective}

Cette définition a été proposée en 1923 par Terzaghi pour les sols saturés. Elle consiste à assimiler la roche saturée à un matériau continu équivalent, à supposer la pression de pore définie en tout point de ce matériau équivalent et à négliger l'influence de cette pression uniforme. Il en résulte que le comportement mécanique du matériau équivalent est simplement une fonction de la contrainte effective $\sigma^{\prime}$ définie par la relation :

$$
\underset{\sim}{\sigma^{\prime}}=\underset{\sim}{\sigma}-\mathrm{P} 1
$$

Si l'on se réfère à l'analyse du paragraphe 2.2 ., on remarque que la contrainte effective n'est autre que la valeur moyenne $(1-f) \sigma^{\prime M}$ et la pression P 1 la valeur moyenne $f \sigma^{\prime F}$. Avec cette notation l'équation d'équilibre du matériau B s'écrit donc :

$$
\operatorname{div} \underline{\sigma}^{\prime}+\operatorname{grad} P+\rho \underline{b}=0
$$

Il a été montré en outre que le comportement élastique (linéaire et non linéaire) du matériau continu B, équivalent à la roche saturée, pouvait également être exprimé en fonction de cette contrainte effective si l'effet de la compressibilité du matériau $\mathrm{M}$, équivalent à la matrice et aux pores non connectés, était négligeable devant la déformation du matériau B.

Finalement il a été proposé que le développement des ruptures fragiles (fissures et microfissures) ne dépendait que de l'état de contrainte $(\sigma-\mathrm{P} 1)$ si la pression interstitielle ne générait pas de concentrations de contraintes (espace des pores entièrement interconnecté, matériau relativement homogène) et ne modifiait pas le comportement rhéologique de la matrice solide (évolution d'un comportement fragile vers un comportement ductile quand la pression hydrostatique augmente ainsi que celà est le cas pour la calcite ou le sel gemme par exemple).

Nous concluerons donc que bien que la notion classique de contrainte effective ne constitue pas une loi physique rigoureuse, elle représente une approximation réaliste lorsque certaines conditions sont satisfaites.

Notons finalement que cette notion de contrainte effective n'est applicable que pour l'étude du comportement global de la roche saturée et qu'elle ne peut, en aucun cas, être confondue avec l'état de contrainte réel dans la matrice solide.

\subsubsection{Contrainte effective et porosité d'aire}

Biot (1955) a proposé d'étudier le comportement d'une roche saturée en considérant les déformations d'un matériau continu $C$ obtenu en supposant que la matrice solide remplit la totalité du volume occupé par la roche saturée mais que la pression du liquide interstitiel n'est définit qu'à l'intérieur de l'espace poreux interconnecté. Ainsi, selon son modèle, le comportement de la roche saturée serait simplement fonction de la contrainte effective :

$$
\stackrel{\sim}{\prime \prime}^{\prime \prime}=\underset{\sim}{\sigma}-a \mathrm{P} \underset{\sim}{1}
$$

où :

$\sigma$ est la contrainte définie pour le matériau équivalent $\mathrm{B}$,

$a$ est la porosité de surface (supposée constante),

$\mathrm{P}$ est la pression hydrostatique dans les pores.

Il a été montré au paragraphe 3.1. que l'équation constitutive dérivée par Biot était équivalente à celle dérivée pour le matériau B ; elle ne s'applique donc pas au matériau C considéré par Biot en 1955 dans son équation d'équilibre et seule sa première étude est donc en accord avec le modèle proposé ici.

Les résultats expérimentaux indiquent tous que la désintégration des roches ne dépend pas de la contrainte effective caractérisée par l'équation (45). Nous conclurons qu'apparemment ce modèle ne peut être appliqué de façon simple à l'étude de la déformation des roches saturées; il devrait donc être abandonné.

\subsubsection{Contrainte effective et porosité limite}

Cette théorie, proposée par Terzaghi en 1945 pour l'étude de la résistance à la compression des roches saturées, est supposée prendre en compte le fait que la porosité d'aire (définie de la façon classique par le rapport des aires occupées par le liquide sur l'aire totale de la surface considérée) d'une surface donnée dépend de la géométrie de cette surface et qu'il existe une surface pour laquelle la porosité d'aire est maximum. ce maximum est appelé porosité d'aire limite.

En effet, même dans le cas d'une porosité isotrope, il est possible de définir une surface pour laquelle la porosité d'aire est supérieure à la porosité de surface telle qu'elle a été définie au paragraphe 2.1 . de ce texte. Considérons, par exemple, un empilement de sphères rigides; une surface qui ne passe que par les vides et les contacts sphères à sphères présente une porosité d'aire bien supérieure à la porosité de surface de ce système.

Ainsi, pour des conditions de chargements données, les contraintes moyennes supportées par la portion solide d'une telle surface sont bien supérieures à celles qui seraient supportées par la fraction solide des surfaces planes. Si l'on suppose que la rupture doive se développer dans les régions où les contraintes moyennes sont les plus grandes, on en déduit que les surfaces de ruptures doivent correspondre à des surfaces à porosité d'aire limite. Pour caractériser la rupture des roches 
saturées, on devrait donc considérer les contraintes effectives définies par la relation :

$$
\tilde{\sigma}^{\prime \prime \prime}=\underline{\sigma}-a^{\prime} \mathrm{P} \underset{\sim}{1}
$$

où $a^{\prime}$ est la porosité d'aire limite.

Les résultats expérimentaux de Robinson (1959) semblaient confirmer cette hypothèse et ont conduit Robinson et Holland (1969) a proposer des valeurs de la porosité d'aire limite variant de 0,6 , pour les roches très faiblement perméables telles que les marnes calcaires, à 1 pour les roches très perméables telles que les grès. De plus, Robinson et Holland ont observé que cette porosité limite augmentait avec la pression de confinement pour devenir quasiment égale à 1 pour toutes les roches lorsque la pression de confinement effective était suffisamment élevée.

Cependant, les résultats expérimentaux de Brace et Martin (1968) ont indiqué que même pour des roches à très faible perméabilité (donc à faible porosité limite) la résistance à la compression dépendait des contraintes effectives classiques telles que définies par Terzaghi en 1923. Cependant, ce point ne peut être vérifié expérimentalement que si la vitesse de déformation utilisée pour les essais triaxiaux drainés, effectués sur ces roches, est suffisamment faible pour éviter le développement de gradient de pression dans le liquide interstitiel (Ladanyi (1970)) du fait de la dilatance.

Or, les essais de Robinson sur le calcaire de l'Indiana, dont les résultats ont été utilisés par Robinson et Holland pour leur vérification expérimentale de la théorie de porosité limite, ont été réalisés à des vitesses de déformations relativement importantes (de l'ordre de $10^{-4} \mathrm{~s}^{-1}$ ) incompatibles avec des conditions de drainage parfait. On peut donc conclure, si l'on considère les résultats expérimentaux exposés au paragrāphe 4.1.2., que la mesure de porosité limite proposée par ces auteurs ne faisait que refléter les variations de dilatance.

Ces résultats expérimentaux indiquent que le développement des ruptures fragiles ne dépend donc pas de la valeur maximum des contraintes moyennes dans la matrice solide mais d'instabilités énergétiques locales dues aux concentrations de contraintes introduites par l'hétérogénéité de la roche. Cette observation met en cause la validité du concept de critères de rupture établis à partir des contraintes moyennes calculées d'après les forces extérieures appliquées sur l'échantillon (notion de courbe intrinsèque par exemple). Etant donné que l'état de contrainte exact en tout point de la matrice ne peut être calculé, seuls des critères de rupture, du type de celui proposé au paragraphe 4.2.3., dérivés de la notion de balance énergétique, devrait permettre d'analyser de façon satisfaisante le développement de la désintégration dans les roches.

Concluons simplement que de toutes les définitions de contrainte effective proposées dans la littérature, seule la définition proposée par. Terzaghi en 1923 est vérifiée expérimentalement ; un raisonnement physique simple a montré cependant que l'hypothèse de Terzaghi n'est valide que si les eífets de la pression hydrostatique appliquée dans l'espace poreux interconnecté est négligeable. Cette théorie ne constitue donc pas une loi physique rigoureuse mais correspond simplement à une simplification pratique.

\subsection{Influence de la vitesse de déformation et des conditions de drainage}

Lorsque l'hypothèse d'un comportement élastique est acceptable et que les conditions d'application de la théorie classique des contraintes effectives sont satisfaites, le processus de déformation d'une roche saturée peut être analysé grâce aux trois équations du système (47) si l'on suppose que le liquide s'écoule selon la loi de Darcy :

$$
\begin{aligned}
& \sigma_{i j, i}^{\prime}+\mathrm{P},{ }_{j}+\rho b_{j}=0 \\
& \varepsilon_{i j}=\left(-\frac{\nu_{B}}{\mathrm{E}_{B}} \mathrm{I}_{1}+b \mathrm{I}_{1}^{2}+c \mathrm{I}_{2}\right) \delta_{i j}+ \\
& \quad+\left(\frac{1+\nu_{B}}{\mathrm{E}_{B}}+e \mathrm{I}_{1}\right) \sigma_{i j}^{\prime}+g \sigma_{i k}^{\prime} \sigma_{k j}^{\prime} \\
& \dot{\mathrm{U}}_{i}-\dot{u}_{i}=\mathrm{K}_{i j}\left(\rho b_{j}-\mathrm{P},{ }_{j}\right)
\end{aligned}
$$

avec :

$$
\begin{aligned}
& \mathrm{I}_{1}=\sigma_{i i}^{\prime}, \mathrm{I}_{2}=\frac{1}{2} \sigma_{i i}^{\prime} \sigma_{j j}^{\prime}(i \neq j) \\
& \sigma_{i j}^{\prime}=\sigma_{i j}-\mathrm{P} \delta_{i j}
\end{aligned}
$$

$\dot{\mathrm{U}}_{i}=$ vitesse du liquide;

$\dot{u}_{i}=$ vitesse du solide;

$\mathrm{K}_{i j}=$ perméabilité de la roche;

$b_{j}=g \delta_{j 3}$ généralement ( $g$ est l'accélération de la pesanteur);

dans le cas de l'élasticité linéaire $b=c=e=g=0$.

La loi de Darcy, ainsi qu'elle est représentée par la troisième équation du système (47), suppose que la perméabilité est indépendante de l'état de contrainte, ce qui n'est généralement pas le cas (Mordecai et Morris (1969) ; Jouanna (1972)), elle n'est valide, en outre, que pour des conditions d'écoulement bien précises.

Ainsi donc, même dans les meilleures conditions d'analyse, la représentation du comportement dynamique des roches saturées reste approximative.

Dès que les conditions de chargement sont telles que des ruptures fragiles se développent, on ne sait plus représenter mathématiquement le comportement d'une roche saturée, étant donné que d'une part la loi de variation de la perméabilité est inconnue et que d'autre part la dilatance irréversible ne peut être modélisée. Pour les roches sèches, on prend en compte le comportement post-élastique de la roche en introduisant dans les calculs (programme d'éléments finis par exemple) une représentation numérique schématique des courbes obtenues expérimentalement (Daemen (1975)).

Pour obtenir de telles courbes dans le cas des roches saturées, deux types d'essais doivent être effectués :

- des essais drainés pour lesquels la pression de pore est maintenue constante (cas des essais précédemment décrits) ;

- des essais non drainés pour lesquels la pression de pore évolue en fonction des variations de volume poreux interconnecté et de la compressibilité du liquide de saturation.

Ces deux conditions extrêmes de drainage permettent en effet de définir des comportements limites encadrant les lois de comportement réels observés pour des conditions de drainage quelconque.

Lorsque la vitesse de variation de volume poreux interconnecté, résultant de la vitesse de chargement imposé, est supérieur à la vitesse d'écoulement du liquide, il devient impossible d'effectuer des essais drainés. On peut alors utiliser l'une des deux méthodes suivantes pour déduire des courbes de déformations drainées fictives. Elles supposent toutes deux que le principe classique de contrainte effective est valide. 
La première méthode consiste à effectuer des essais non drainés au cours desquels la pression de confinement varie de la même quantité que la pression interstitielle, de sorte que la pression de confinement effective reste constante. Cette technique, qui à notre connaisn'a jamais été appliquée, devrait permettre d'étudier l'influence des vitesses de déformation sur le comportement de la roche saturée sans être gêné par l'effet de drainage partiel précédemment décrit; il permettrait ainsi de préciser les effets physicochimiques d'interaction solide-liquide.

La deuxième méthode consiste à effectuer des essais non drainés classiques, c'est-à-dire à maintenir la pression de confinement constante mais à effectuer les essais à partir d'un certain nombre de pressions de confinement effectives initiales différentes.

Si l'on suppose que la déformation d'une roche saturée est indépendante du chemin de chargement, on peut alors déduire de ces essais des courbes fictives correspondant à des essais drainés en joignant les points de diverses courbes pour lesquels la pression de confinement effective est la même (voir fig. 13). Toutefois, cette approche n'est pas très satisfaisante car dans le domaine post-élastique, pour une même pression de confinement effective ponctuelle, le degré de microfissuration dépendra du chemin de chargement parcouru ainsi que cela est indiqué ci-après.

Les figures 14,15 et 16 représentent l'influence des conditions de drainage sur le comportement d'une roche saturée. Sur la figure 14, par exemple, on notera que, pour des conditions non drainées, la déformation axiale moyenne $\left(\mathrm{B}_{u}\right)$ qui correspond à la charge axiale moyenne pour laquelle la pression interstitielle reprend sa valeur originale, est plus grande que celle obtenue pour la même pression de confinement effective en conditions drainées (point $\mathrm{B}_{d}$ ). Etant donné que, pour les conditions non drainées, avant que ce point de déformation ne soit atteint, la pression de confinement effective est plus faible que celle que l'on aurait en condition drainée, la densité de microfissures est plus grande et, par conséquent, la déformation correspondante est plus importante.

En raisonnant de façon similaire, on peut montrer que la transition classe I-classe II est fortement influencée par les conditions de drainage. Ainsi, par exemple, dans le cas de la dilatance positive, on observe l'effet classique de renforcement apparent (écrouissage) dû à la diminution de pression interstitielle, tandis que dans le cas de la dilatance négative, on observe l'effet, moins connu, d'affaiblissement apparent (contre écrouissage) dû à l'augmentation de pression de pore. Il est

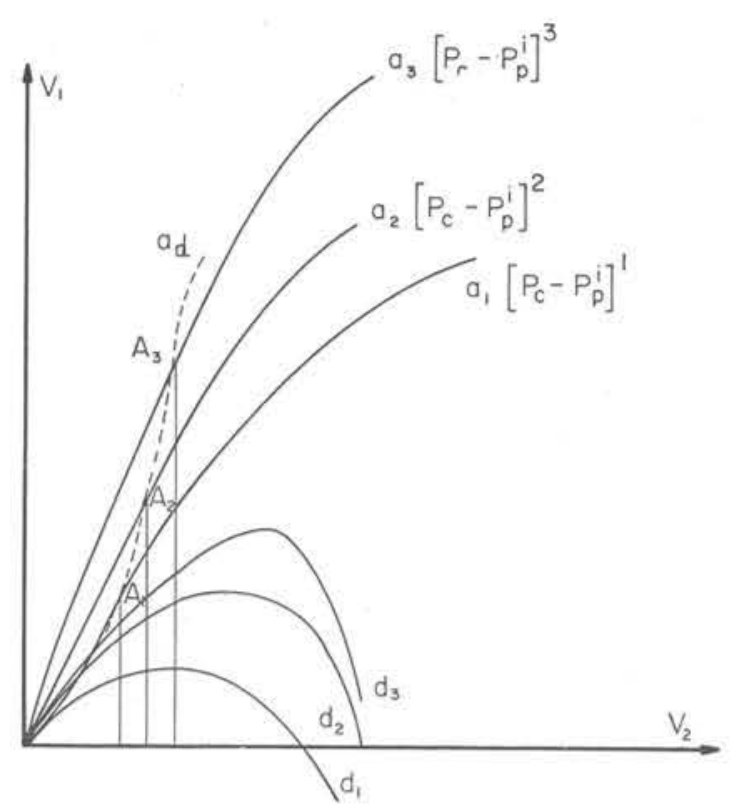

Fig. 13. - Obtention des courbes de réponses pour des conditions de drainage parfait à partir d'essais triaxiaux non drainés :

- courbes a : variations de la grandeur $V_{1}$ en fonction de la grandeur $V_{2}$ pour différentes conditions de pression de confinement effective initiales $\left(\mathrm{P}_{\mathrm{c}}-\mathrm{P}_{\mathrm{p}}\right)$ (indices $\left.1,2,3\right)$;

- courbes d: variations de la pression interstitielle en fonction de la grandeur $\mathrm{V}_{2}$.

Les points $A_{1}, A_{2}, A_{3}$ correspondent à une valeur identique de la pression de confinement effective $\left(\mathrm{P}_{\mathrm{c}}-\mathrm{P}_{\mathrm{p}}\right)$.

évident que le comportement en conditions de drainage quelconque s'écartera d'autant plus du comportement en conditions drainées que la dilatance sera importante et que la vitesse d'écoulement du fluide sera plus faible vis-à-vis de la vitesse de déformation de la roche saturée.

Le même type de raisonnement peut d'ailleurs s'appliquer aux déformations par cisaillement direct ainsi que cela est schématiquement représenté figure 17 . On observera que les conditions de variations de pression de pore dans les blocs influencent le comportement apparent du joint du fait qu'elles influencent la contrainte normale effective. 


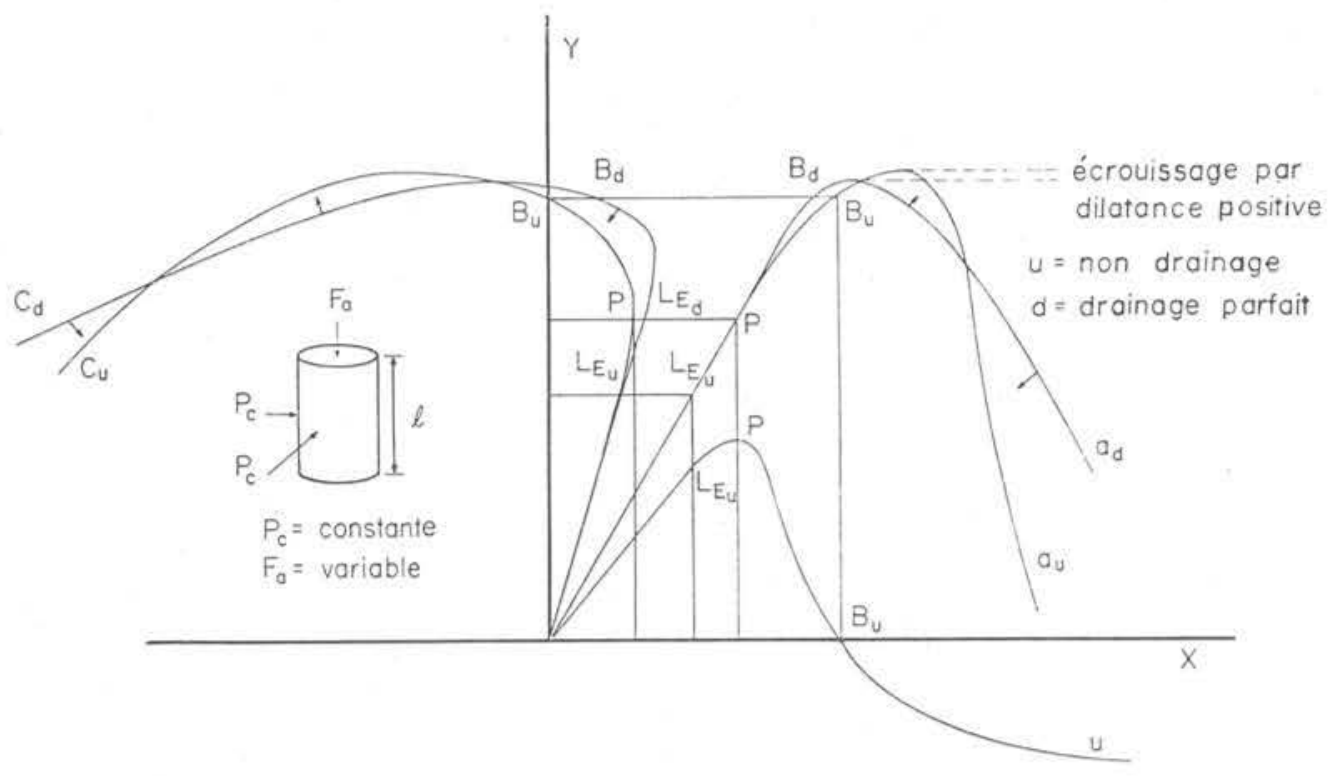

Fig. 14.

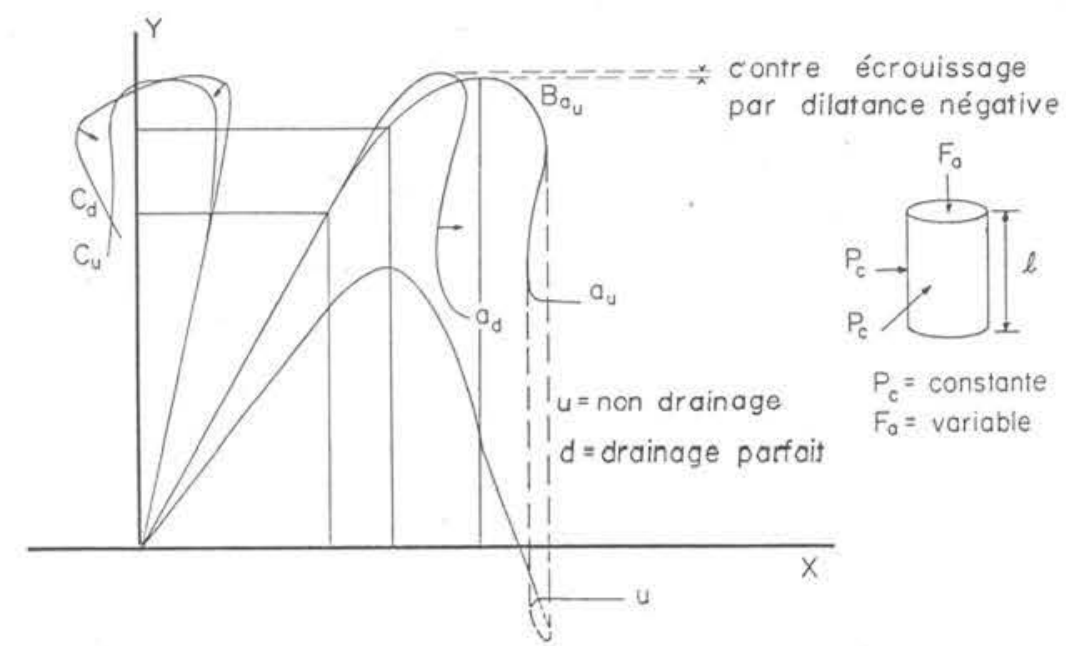

Fig. 15

Fig. 16.

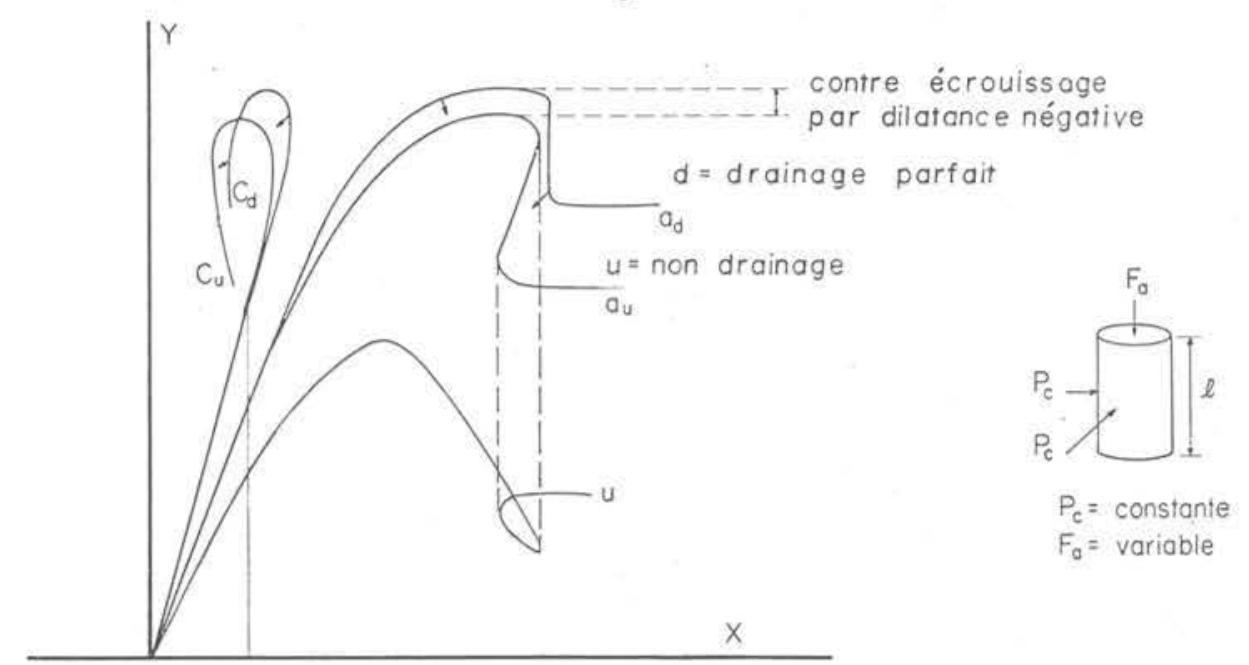

Fig. 14 à 16. - Comparaison des essais triaxiaux drainés et non drainés :

- courbes $\mathrm{a}_{\mathrm{d}}, \mathrm{d}_{\mathrm{u}}$ : axe des $\mathrm{x}$ : déformation axiale moyenne;

axe des y : variations de la charge axiale moyenne;

- courbes $c_{d}, c_{u}$ : axe des $x$ : variations de volume poreux interconnecté par unité de volume global ;

axe des $y$ : variations de la charge axiale moyenne;

: axe des $\mathrm{x}$ : déformation axiale moyenne;

axe des $\mathrm{y}$ : variation de pression interstitielle pour l'essai non drainé

$\mathrm{P}$ : maximum de la pression interstitielle. 

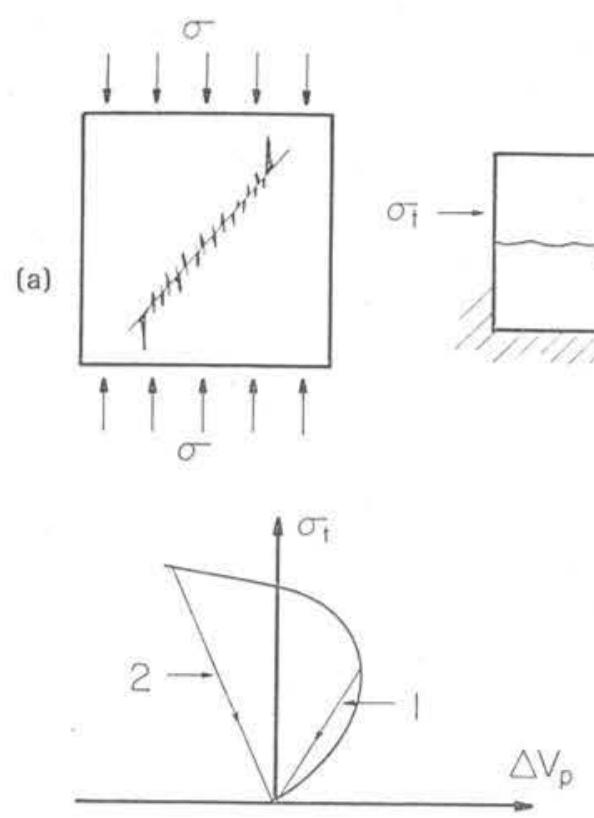
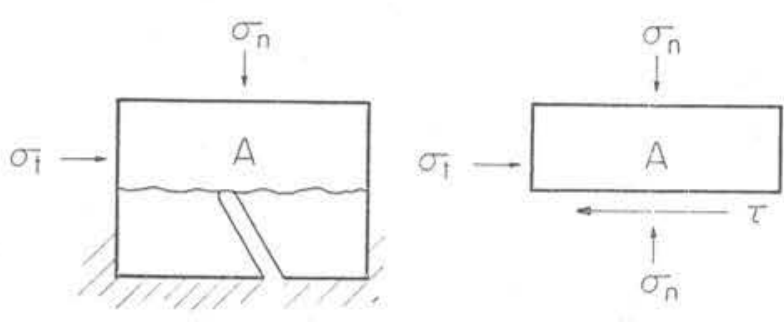

$(b, c)$

L'essai de cisaillement direct

I. si $\sigma_{1} \backslash, \Delta V_{p}$,

2. si $\sigma_{1} \backslash, \Delta V_{p} \backslash$

$\Delta V_{p}=$ variation de volume poreux dans le bloc A

Fig. 17. - Effet de la dilatance sur l'essai de cisaillement direct non drainé : (a) glissement le long d'une fente inclinée;

(b, c) essai de cisaillement direct.

\section{CONCLUSION}

Cette étude a montré qu'il était possible d'étudier le comportement mécanique des roches saturées en supposant que tout point de l'espace occupé par la roche est en partie solide et en partie liquide.

Lorsque le comportement de la matrice solide est linéairement élastique, que celui du matériau continu équivalent à la roche saturée est élastique (linéaire ou non-linéaire) et que le liquide interstitiel s'écoule selon la loi de Darcy, les déformations d'une roche saturée peuvent être analysées de façon rigoureuse si l'on suppose que les variations de perméabilité en fonction de l'état de contrainte sont négligeables ou connues.

Lorsque les conditions de chargement sont telles que des ruptures fragiles se produisent (fissures au micro- fissures), seule une étude expérimentale permet de préciser le comportement mécanique des roches fragiles et saturées. Il semble que la meilleure façon de procéder pour cette caractérisation consiste à effectuer des essais triaxiaux de compression (éventuellement polyaxiaux) sur deux séries d'éprouvettes saturées placées sous conditions drainées pour les unes et non-drainées pour les autres. Si le principe des contraintes effectives est valide, il est alors possible de prendre en compte le phénomène de dilatance (positive et négative) en caractérisant le comportement de la roche saturée par une série de courbes schématiques correspondant aux conditions limites de drainage.

\section{REMERCIEMENTS}

Cet exposé résume une partie du travail que j'ai effectué pour l'obtention d'un $\mathrm{Ph}$. D. à l'Université du Minnesota. Que le Professeur C. Fairhurst, mon Directeur de thèse, trouve ici l'expression de mes sincères remerciements pour son soutien et ses encouragements durant la réalisation de ce travail. 


\section{REFERENCES}

[1] BARON (G.), CASTEL (Y.) and HABIB (P.). «Influence de la pression interstitielle sur les caractéristiques mécaniques des roches en condition de fond » ; Revue I.F.P., Vol. XVIII, No hors série (1963).

[2] BIOT (M.A.). - " Général theory of three dimensional consolidation »; Jour. App. Phys., Vol. 12, p. 155-164 (1941).

[3] BIOT (M.A.). - « Theory of elasticity and consolidation for a porous anisotropic solid "; Jour. App. Phys., Vol. 26, p. 182 (1955).

[4] BRACE (W.F.), PAULDING JR (B.W) and SCHOLZ (C.). - - Dilatancy in the fracture of crystalline rocks "; Jour. Geophys. Res., Vol. $71, \mathrm{~N}^{\circ} 16$, p. $3939-3952$ (1966).

[5] BRACE (W.F.) and MARTIN III (R.J.). "A test of effective stress law for crystalin rocks of low porosity "; Int. Jour. Rock Mech. Min. Sc., Vol. 5, p. 415 (1968).

[6] CORNET (F.H.). - «Comparative Analysis by the displacement-discontinuity method of two energy criteria of fracture ", soumis pour publication à Int. Jour. Eng. S-c. (1976).

[7] CROUCH (S.L.). - « Solution of plane elasticity problems by the displacement-discontinuity method "; à paraître dans Int. Jour. Numerical Methods in Engineering, Vol. 10, p. 301-343 (1976).

[8] DAEMEN (J.J.K.). - " Tunnel Support Loading Caused by Rock Failure »; Technical Report M.R.D. 3-75. Missouri River Division, Corps of Engineers, Omaha (1975).

[9] EDMUND (J.M.) and PATERSON (M.S.). "Volume changes during the deformation of rocks at high pressures ": Int. Jour. Rock Mech. Min Sc., Vol. 9, p. 161-182 (1971).

[10] FREUDENTHAL (A.M.), - « Constitutive equations of Rock with shear dilatancy ». Final technical Report. The George Washington Univ. School of Engineering and App. Sc., Washington D.C. (1975).

[11] GEERTSMA (J.). - «The effect of fluid pressure decline on volumetric changes of porous rocks »; Petr. Trans. Am. Ins. Min. Eng., Vol. $210, N^{\circ} 12$, p. $331-339$ (1957).

[12] GREEN (A.E.), STEEL (T.R.). - « Constitutive equation for interacting continua"; Int. Jour. Eng. Sc., Vol. 4, p. $483-501$ (1966).

[13] GRIFFITH (A.A.). - " The phenomenon of rupture and flow in solids"; Phil. Trans. Roy. Soc., London, A 221 (1921).

[14] HADLEY (K.). - «Azimuthal variation of dilatancy »; Jour. Geophys. Res., Vol. 80, № 35, p. 4845 (1975).

[15] HANDIN (J.), HAEGER (R.V.), FRIEDMAN (M.) and FEATHER (J.N.) - « Experimental deformation of sedimentary rocks under confining pressure: pore pressure tests »; Bull. Am. Ass. Petrol. Geol., Vol. 47, No 5, p. 717 (1963).
[16] HOAGLAND (R.G.), HAHN (G.T.), ROSENFIELD (A.R.) and SIMON (R.). - «Influence of microstructure on fracture propagation in rock »; final A.R.P.A. report contract $\mathrm{N}^{\circ}$ H0210006 (U.S.B.M. Twin Cities) (1972).

[17] HUDSON (J.A.), BROWN (E.T.) and FAIR. HURST (C.). - "A method of optimizing the control of rock failure in servo controlled laboratory tests »; Rock Mech., Vol. 3, No 4, p. 217-224 (1970).

[18] JOUANNA (P.). - - Essais de percolation au laboratoire sur des échantillons de micaschistes soumis à des contraintes »; Percolation through fissured rock, Symp. Int. Rock Mech., Stuttgart, Wittke editor (1972).

[19] LADANYI (B.). - « Comments on Brace and Martin paper ». Int. Jour. Rock Mech. Min, Sc. Vol. 7 (1970).

[20] LUBINSKI (A.). - " The theory of elasticity for porous bodies displaying a strong pore structure » ; Proc. Sec. Nat. Cong. App. Mech., p. 247 (1954),

[21] MORDECAI (M.) and MORRIS (L.H.). - " An investigation into the change of permeability occuring in a sandstone when failed under triaxial stress conditions "; 12th Symp. Rock Mec. Univ. Missouri, Rolla, A.I.M.E., Clark editor (1971).

[22] MORLIER (P.). - « Comportement mécanique des solides poreux - Domaine élastique des corps poreux - Rôle de la pression de pore »; Revue de l'Industrie Minérale, $\mathrm{N}^{\circ}$ spécial, 15 décembre 1970.

[23] NUR (A.) and BYERLEE (J.D.). - " An exact effective stress law for elastic deformation of rocks with fluids " ; Jour. Geophys. Res., Vol. 76, p. 6414 (1971).

[24] RICE (J.R.). - «On the stability of dilatant hardening for saturated rock masses "; Jour. Geophys. Res., Vol. 80, No 11, p. 1531 (1975).

[25] ROBINSON (L.H.). - " The effect of pore and confining pressure on the failure process in sedimentary rocks " ; Colorado School Mines, quart., Vol. 54, No 3, p. 177-199 (1959).

[26] ROBINSON (L.H.) and HOLLAND (W.E.). "Some interpretation of pore fluid effects in rock failure »; 11th Symp. Rock Mech., Berkeley, A.I.M.E., Sommerton editor (1969).

[27] RUDNIKI (J.W.) and RICE (J.R.). - « Conditions for the localization of deformation in pressure-sensitive dilatant materials »; Brown University, Div. of Eng., N.S.F. Geophys. Program., Grant G.A. - 43 380, Report № 2 (1974).

[28] RUMMEL (F.) and FAIRHURST (C.). - Determination of the post failure behavior of brittle rock using a servo controlled testing machine » ; Rock Mech., Vol. 2, p. 189-204 (1970).

[29] SCHEIDEGER (A.E.). - " The physics of flow through porous media "; revised edition, The Macmillan comp., N.Y. (1964). 
[30] SCHOCK (R.N.), HEARD (H.C.) and STEPHENS (D.R.). - « Stress-strain behavior of a granodiorite and two graywacks on compression to 20 kilobars "; Jour. Geophys. Res., Vol. 78, No 26, p. $5922-5941$ (1973).

[31] STUART (W.D.) and DIETRICH (J.). - " Continuum theory of rock dilatancy » ; 3rd Int. Congr. Rock Mech., Theme 2, Denver, Int. Soc. Rock Mech. (1974).

[32] TERZAGHI (K.). - « Die Berechnung der durchlassingkeitsziffer des tones aus den verlauf des hydrodynamishen Spannungserscheinungen »;
Sitz. Akid. Wissen, Wien Mat. Naturwiss. K1. Ab. 2A, 132, p. 105-124. (1923).

[33] TERZAGHI (K.). - « Stress conditions for the failure of saturated concrete and rock "; Proc. Am. Soc. Test. Mat., Vol. 45, p. 777-801 (1945).

[34] TRUESDELL (C.). - * The mechanical foundations of elasticity and fluid dynamics "; Jour. Rational Mechanics, Vol. 1, p. 173-197 (1952).

[35] WAWERSIK (W.R.). - « Detailed analysis of rock failure in laboratory compression experiments "; Ph. D. Thesis, unpublished, Univ. Minn. (1968). 